\title{
On Real Cyclic Sextic Fields
}

\author{
By V. Ennola, S. Mäki and R. Turunen
}

\begin{abstract}
A table of units and class numbers of real cyclic sextic fields with conductor $f_{6} \leqslant 2021$ has been given by the second author [13]. We first fill in the gaps in [13] and then construct an extended table for $2021<f_{6}<4000$. The article contains results about Galois module structure of the unit group, relative norms of the units, and ideal classes of the subfields becoming principal in the sextic field. The connection with Leopoldt's theory [11] is described. A parametric family of fields containing exceptional units [14] is constructed. We give statistics referring to class numbers of fields with prime conductor, the appearance of units of different types if the relative class number is $>1$, Leopoldt's unit index, and the signature rank of the unit group.
\end{abstract}

1. Introduction. A table of units and class numbers of the 1337 real cyclic sextic fields $K_{6}$ with conductor $f_{6} \leqslant 2021$ has been given by the second author [13]. In this table there are 12 gaps (included in the cardinality 1337), the reason for the failure being in 6 cases similar gaps in M.-N. Gras's table of cyclic cubic fields [5], and in the 6 other cases, the appearance of too large numbers which the program could not handle. Since it seems to be of importance for many purposes to have a complete result reaching as far as possible, we have taken up the work and have constructed an extended table of the 1743 fields $K_{6}$ with $2021<f_{6}<4000$. M.-N. Gras's table of real cyclic quartic fields, [6] and [7], and the main table in [5] also have the same range, i.e., conductor $<4000$, but in [3] the first and third author have obtained the result in the cubic case up to conductor $<16000$.

The efficient multiprecision routines developed in [2] and [3] enabled us first to fill in the gaps in [5] and then complete the computations in the 12 open sextic cases as well as in other difficult large cases. Hence, the table in [13] and the new one together provide a complete answer for all real cyclic sextic fields with conductor $<4000$.

The gaps in [5] have independently been filled in by Godwin [4] whose method is entirely different from ours, the latter being based on the same adaptation of the Voronoi algorithm we used in [2].

The present paper should be regarded as a supplement to [13]. We use the same terminology and notation with one exception: the ambiguous term " $f_{6}$ is decomposable" is replaced by the more accurate " $\chi_{6}$ is decomposable". See Section 2 for an explanation and for a list of additional notation.

In Section 3 we make a comparison between the terminology in [13] and the one of Leopoldt [11] applicable to any real abelian field. Although the latter is unnecessarily complicated in the cyclic case it has also been used there (e.g., in [6] and [7]) for the sake of uniformity. In addition, we give some supplementary remarks to [13].

Received August 8, 1984.

1980 Mathematics Subject Classification. Primary 12-04, 12A35, 12A45, 12A50. 
Section 4 contains a description of the unit group as Galois module. This module structure is easily derived from known results about integral representations of the Galois group.

In the computations an important part is played by the relative norms of the unit $\xi_{A}$ defined in [13]. In Section 5, we obtain rather precise information about these norms by a simple argument.

The next three sections are concerned with capitulation of ideal classes, i.e., nonprincipal ideal classes of subfields becoming principal in the sextic field. In the real cyclic quartic case the analogous question has been investigated by M.-N. Gras [6], [7]. For the quadratic and cubic subfield separately we prove practical criteria by means of which we have been able to verify that such a capitulation takes place for $f_{6}<4000$, provided that certain natural necessary conditions are satisfied. In the course of the investigation we need elements of $K_{6}$ satisfying Hilbert 90 for either subfield and for various units of $K_{6}$. In particular, for the quadratic subfield and for a generating relative unit $\xi_{R}$ we define in a natural way such an element denoted by $\omega$. An examination of the possibility $\omega=0$ incidentally leads to a parametric family of fields $K_{6}$ containing exceptional units (in the sense of Nagell [14]) which do not belong to any of the proper subfields. The family can also be chosen so that four independent units have parametric representations but we have not been able to find such a representation for a missing (quadratic) unit.

In the new table the fields are listed in practically the same format as in [13]. The slight format changes are indicated at the outset of Section 9. The bulk of Section 9 consists of statistics. We first give a list of all fields $K_{6}$ such that the conductor $f_{6}$ is a prime $<4000$ and the class number $h_{6}>1$. Next we divide all fields $K_{6}$ with relative class number $h_{R}>1$ into 16 different types depending on the existence of units of different kinds, and we give the frequencies of fields belonging to each type. The fields belonging to scarce types are identified explicitly. The ensuing table contains statistics referring to the signature ranks for $\chi_{6}$ decomposable or nondecomposable separately. In the next table we have gathered all cases in which the norm-positive cubic units are totally positive. A knowledge of these cases is of importance, e.g., in questions concerning the signature rank. Further, we consider the distribution of the values of Leopoldt's unit index $Q_{K}$. We conclude the paper by indicating a frequently occurring connection between the relative class numbers of distinct fields $K_{6}$ having the same conductor and the same quadratic or cubic subfield.

The bulk of the program is constructed for the UNIVAC 1108 system using FORTRAN V programming language. Some auxiliary computations have also been done on the DEC-20 computer. We express our appreciation for the cooperation we have received from the members of the staff of the Computer Centre at the University of Turku. We are particularly obliged to Mr. Jussi Salmela, M. Sc., for his generous help, and to Mrs. Marjo Henriksson for writing the data on a magnetic tape. The work has been supported financially by the Academy of Finland.

2. Notation. The present paper is a continuation of [13] and we use exactly the same terminology and notation throughout with one exception. The term "conductor $f_{6}$ is decomposable", adapted directly from [9, p. 60], is replaced by "the character 
$\chi_{6}$ is decomposable" used, e.g., in [11]. The reason for this is the following annoying fact. Let $p$ be either 9 or a prime $\equiv 7 \bmod 12$. Then, in the old terminology the sextic conductor $f_{6}=8 p$ is decomposable if $f_{2}=8$, but nondecomposable if $f_{2}=$ $8 p /(p, 3)$. In both cases $f_{3}=p$, and there are exactly 25 such values of $p<500$. On the other hand, it is easily seen that these values of $f_{6}$ are the only ones for which such a dubious situation occurs.

First, we recall from [13] some of the most common notations. For $n \in\{1,2,3$, $6\}, K_{n}$ is a real cyclic extension of degree $n$ over $\mathbf{Q}$ having conductor $f_{n}$, class number $h_{n}$, ring of integers $\mathcal{O}_{n}$, and unit group $U_{n}$. We write $f_{2}=m$ or $4 m$, where $m$ is a square-free integer, and $f_{3}=\left(a^{2}+3 b^{2}\right) / 4$, where $a$ and $b$ satisfy the normalization conditions [13, p. 6, (3)] introduced by Hasse [9]. $S_{n / q}$ and $N_{n / q}$ are the trace and norm from $K_{n}$ to $K_{q} . G=\langle\sigma\rangle$ is the Galois group of $K_{6}$, and the conjugates of a number $\gamma \in K_{6}$ are $\gamma, \gamma^{\prime}=\gamma^{\sigma}, \gamma^{\prime \prime}=\gamma^{\sigma^{2}}$, etc. The fundamental unit of $K_{2}$ is denoted by $\mu$, and $\tau$ is a norm-positive cubic unit such that $\left\langle-1, \tau, \tau^{\prime}\right\rangle=U_{3}$. Finally, $U_{R}=\left\{\varepsilon \in U_{6} \mid N_{6 / 3}(\varepsilon)= \pm 1, N_{6 / 2}(\varepsilon)= \pm 1\right\}$ is the group of relative units, and $\xi_{R}$ is a generating relative unit, i.e., $\left\langle-1, \xi_{R}, \xi_{R}^{\prime}\right\rangle=U_{R}$.

If $a$ is an integral ideal of any of the rings $\mathcal{O}_{n}$ then divisibility by a and congruence modulo a are defined in a natural way in the ring consisting of the numbers of the field $K_{n}$ representable as a quotient of numbers of $\mathcal{O}_{n}$ with denominator prime to a. In particular, if $\mathfrak{p}$ is a prime ideal and $\gamma$ is $\mathfrak{p}$-integral, then $\mathfrak{p}^{k} \| \gamma$ and $\nu_{\mathfrak{p}}(\gamma)=k$ both mean that $\gamma \equiv 0 \bmod \mathfrak{p}^{k}, \gamma \neq 0 \bmod \mathfrak{p}^{k+1}$.

The cyclotomic field $\mathbf{Q}\left(\zeta_{k}\right)=\mathbf{Q}(\exp (2 \pi i / k))$ is denoted by $C(k)$. In Section $4, R$ denotes the integral group ring $\mathbf{Z}[G]$. For any subgroup $U$ of $U_{6}$ we write $|U|=$ $\{|\varepsilon| \mid \varepsilon \in U\}$. The unit index from Leopoldt [11] is defined as $Q_{K}=\left[U_{6}: U_{2} U_{3} U_{R}\right]$ and it is easy to see that

$$
Q_{K}=\left\{\begin{aligned}
12 & \text { if } N_{6 / 2}\left(U_{6}\right)=U_{2} \text { and }\langle-1\rangle N_{6 / 3}\left(U_{6}\right)=U_{3}, \\
3 & \text { if } N_{6 / 2}\left(U_{6}\right)=U_{2} \text { and }\langle-1\rangle N_{6 / 3}\left(U_{6}\right) \neq U_{3}, \\
4 & \text { if } N_{6 / 2}\left(U_{6}\right) \neq U_{2} \text { and }\langle-1\rangle N_{6 / 3}\left(U_{6}\right)=U_{3}, \\
1 & \text { if } N_{6 / 2}\left(U_{6}\right) \neq U_{2} \text { and }\langle-1\rangle N_{6 / 3}\left(U_{6}\right) \neq U_{3} .
\end{aligned}\right.
$$

In Sections 6, 7, 8 we shall assume that $N_{6 / 2}\left(\xi_{R}\right)=1$ which can be achieved by changing the sign of $\xi_{R}$ if need be. This condition is not necessarily satisfied in the tables. We write $\xi_{R}=\alpha+\beta \sqrt{m}$, where $\alpha, \beta \in K_{3}$. We take $\omega=\tau^{-1}+\xi_{R} \tau^{\prime \prime-1}+$ $\xi_{R}^{\prime} \tau^{\prime-1}$. For $n=2$ or $3, U_{R n}=\left\{\varepsilon \in U_{6} \mid N_{6 / n}(\varepsilon)=1\right\}, \mathrm{Cl}_{n}(\mathrm{c})$ is the ideal class of $K_{n}$ containing a given nonzero fractional ideal $\mathfrak{c}$, and $\mathscr{C}_{6 / n}=\left\{\mathrm{Cl}_{n}(\mathrm{c}) \mid \mathfrak{c} \mathcal{O}_{6}=\gamma \mathcal{O}_{6}\right.$ for some $\left.\gamma \in K_{6} \backslash\{0\}\right\}$ is the group of ideal classes of $K_{n}$ becoming principal in $K_{6}$.

For any other undefined notations appearing in the text one should consult [13, pp. 196-198].

3. Connection with Leopoldt's Theory. For a unified theory of units and class numbers of real abelian fields, the concepts introduced by Leopoldt [11] are indispensable. In the cyclic case the situation is much less complicated, and it is enough to apply Satz 9 in Hasse [10, p. 40] as was also done in [13]. Anyway, it is useful to have an explicit list giving the translation of the basic concepts in [11] into 
our notation as follows:

\begin{tabular}{|c|c|c|}
\hline Einheitengruppe von $\mathbf{K}=K_{6}$ & $\mathbf{E}_{K}$ & $U_{6}$ \\
\hline$\tilde{\chi}_{n}$-Relativeinheiten in $\mathbf{K}$ & $\mathbf{E}_{\tilde{\chi}_{n}}^{\mathbf{K}}=\mathbf{E}_{\tilde{\chi}_{n}}^{+}$ & $\begin{array}{ll}U_{R} & \text { if } n=6 \\
U_{n} & \text { if } n=2 \text { or } 3\end{array}$ \\
\hline Einheitenkern von $\mathbf{K}$ & $\mathbf{E}^{\mathbf{K}}=\mathbf{E}^{\mathbf{K}}$ & $U_{2} U_{3} U_{R}$ \\
\hline Einheitenindex & $Q_{\mathbf{K}}=Q_{\mathbf{K}}^{+}$ & $Q_{K}$ defined in Section 2 \\
\hline Grenzindex & $Q_{(H)}$ & 12 \\
\hline $\begin{array}{l}\text { Erzeugende } \\
\tilde{\chi}_{n}-\text { Relativkreiseinheit }\end{array}$ & $\mathrm{H}_{\bar{\chi}_{n}}$ & $\begin{array}{ll}\eta^{(1+\sigma)\left(1-\sigma^{3}\right)} & \text { if } n=6 \\
\eta_{n} & \text { if } n=2 \text { or } 3\end{array}$ \\
\hline Formaler Kreiseinheitenkern & $\mathbf{H}^{\mathrm{K}}$ & $Y_{6}^{*}$ \\
\hline Klassenzahlkomponenten & $h_{\tilde{\chi}_{n}}$ & $\begin{array}{ll}h_{R}^{*} & \text { if } n=6 \\
h_{n} & \text { if } n=2 \text { or } 3\end{array}$ \\
\hline
\end{tabular}

In this table, $\tilde{\chi}_{n}$ denotes the Frobenius division of the character group of $K_{6}$ containing $\chi_{n}$; a generating character of the field $K_{n}(n=2,3,6), Y_{6}^{*}$ and $h_{R}^{*}$ are new notations introduced here; and $\eta_{n}$ is the cyclotomic unit of $K_{n}\left(\eta=\eta_{6}\right)$ as defined in [10, p. 25].

From $[10$, p. 40$]$ and $[13$, p. 58], we have the central result

$$
h_{6}=h_{2} h_{3} h_{R}=\left[U_{6}: Y_{6}\right]
$$

where $Y_{6}=\left\langle-1, \eta_{2}, \eta_{3}, \eta_{3}^{\prime}, \eta, \eta^{\prime}\right\rangle$ is the group of cyclotomic units in the sense of Hasse. By definition [11, p. 39],

$$
Y_{6}^{*}=\left\langle-1, \eta_{2}, \eta_{3}, \eta_{3}^{\prime}, \eta^{(1+\sigma)\left(1-\sigma^{3}\right)}, \eta^{\left(\sigma+\sigma^{2}\right)\left(1-\sigma^{3}\right)}\right\rangle .
$$

In accordance with [11, p. 41, Satz 20], we have

$$
\left[U_{6}: U_{2} U_{3} Y_{6}\right]=h_{R}, \quad\left[U_{2} U_{3} Y_{6}: U_{2} U_{3} Y_{6}^{*}\right]=12, \quad\left[U_{2} U_{3} Y_{6}^{*}: Y_{6}^{*}\right]=h_{2} h_{3},
$$

where the second equality can be deduced by direct computation, and the other equalities follow from (3.1) and the theorem of Hasse cited above. From [11, p. 40, Eq. (7)], we now have

$$
h_{R}^{*}=\left[U_{2} U_{3} U_{R}: U_{2} U_{3} Y_{6}^{*}\right]=12 h_{R} / Q_{K} .
$$

In [13, p. 17; cf. also p. 59] the second author introduced the group $U_{6}^{*}=U_{2} U_{3} U_{R} Y_{6}$. The index $\left[U_{6}: U_{6}^{*}\right]=1,3,4$ or 12 depending on the existence of certain units $\xi_{B}$, $\xi_{C}$. In order to compute the value of $h_{R}$ from (3.2) one needs the index of $U_{2} U_{3} Y_{6}$ in $U_{6}^{*}$. It is not hard to infer from the considerations in [13] that, in fact,

$$
\left[U_{6}^{*}: U_{2} U_{3} Y_{6}\right]=2^{2 n}\left(K^{2}+K L+L^{2}\right) \text {, }
$$

where $n, K, L$ are determined by $\xi_{1}^{2^{n}}= \pm \xi_{0}, \xi_{1}= \pm \xi_{R}^{K} \xi_{R}^{\prime L}$.

4. The Unit Group as $G$-Module. Let $R=\mathbf{Z}[G]$. The indecomposable $R$-modules have been computed by A. Matuljauskas [12], and, more generally, the question of integral representations of a cyclic group of square-free order has been investigated by J. H. Oppenheim in his thesis [15].

Let us first consider the $R$-module $M=R /\left(1+\sigma+\cdots+\sigma^{5}\right) R$. Write $M_{0}=$ $\left\{x \in M \mid(1+\sigma)\left(1+\sigma+\sigma^{2}\right) x=0\right\}$ and $M_{1}=M / M_{0}$. It is easy to see that

$$
\begin{aligned}
& M_{0}=\left(1-\sigma+\sigma^{2}\right) M \cong R /(1+\sigma) R \oplus R /\left(1+\sigma+\sigma^{2}\right) R, \\
& M_{1} \cong R /\left(1-\sigma+\sigma^{2}\right) R .
\end{aligned}
$$


Note that compared with Oppenheim [15], we have interchanged his $s_{0}$ and $s_{1}$ which causes a change in the definition of $M_{0}$ and $M_{1}$, but the main results remain unaffected. The module $M$ is an extension of $M_{1}$ by $M_{0}$. Let $\Lambda: R \times M_{1} \rightarrow M_{0}$ be a cocycle corresponding to this extension as defined in $[15$, p. 11]. If $\lambda \in$ $\operatorname{Hom}_{\mathbf{z}}\left(M_{1}, M\right)$ satisfies the condition $p \circ \lambda=1$, where $p: M \rightarrow M_{1}$ is the natural projection, one can take

$$
\Lambda(r, x)=r \lambda(x)-\lambda(r x) \text { for } r \in R, x \in M_{1} .
$$

If $\Lambda$ is defined by (4.1), but with $\lambda \in \operatorname{Hom}_{\mathbf{z}}\left(M_{1}, M_{0}\right)$, it is termed a coboundary. Then $\operatorname{Ext}_{R}^{1}\left(M_{1}, M_{0}\right)$ is the factor group cocycles modulo coboundaries. From [15, Corollary 3.17 and Theorem 4.1] it follows that

$$
\begin{aligned}
\operatorname{Ext}_{R}^{1}\left(M_{1}, M_{0}\right) \cong & \operatorname{Hom}_{R}\left(M_{1} /(1+\sigma) M_{1},\left(1+\sigma+\sigma^{2}\right) M_{0} /\left(1+\sigma^{2}+\sigma^{4}\right) M_{0}\right) \\
& \oplus \operatorname{Hom}_{R}\left(M_{1} /\left(1+\sigma+\sigma^{2}\right) M_{1},(1+\sigma) M_{0} /\left(1+\sigma^{3}\right) M_{0}\right) .
\end{aligned}
$$

Let $\left(f_{\Lambda}, g_{\Lambda}\right)$ be the image of the class represented by the cocycle $\Lambda$ under this isomorphism. We have

$$
\begin{aligned}
& M_{1} /(1+\sigma) M_{1} \cong\left(1+\sigma+\sigma^{2}\right) M_{0} /\left(1+\sigma^{2}+\sigma^{4}\right) M_{0} \cong G F(3), \\
& M_{1} /\left(1+\sigma+\sigma^{2}\right) M_{1} \cong(1+\sigma) M_{0} /\left(1+\sigma^{3}\right) M_{0} \cong G F(4),
\end{aligned}
$$

the action of $G$ on the finite fields being defined in an obvious way. It follows from the results of Oppenheim that, for any $x \in M_{1}$,

$$
\left\{\begin{array}{l}
f_{\Lambda}\left(x+(1+\sigma) M_{1}\right)=(1-\sigma)\left(1+\sigma^{2}+\sigma^{4}\right) \lambda(x)+\left(1+\sigma^{2}+\sigma^{4}\right) M_{0} \\
g_{\Lambda}\left(x+\left(1+\sigma+\sigma^{2}\right) M_{1}\right)=(1-\sigma)\left(1+\sigma^{3}\right) \lambda(x)+\left(1+\sigma^{3}\right) M_{0} .
\end{array}\right.
$$

We can define $\lambda$ by

$$
\lambda\left(u+v \sigma+\left(1-\sigma+\sigma^{2}\right) R\right)=u+v \sigma+\left(1+\sigma+\cdots+\sigma^{5}\right) \mathbf{Z} \text { for } u, v \in \mathbf{Z} .
$$

From (4.2) we see that $f_{\Lambda}$ and $g_{\Lambda}$ are both nonzero. By [15, pp. 24-25], $M$ is indecomposable and, on the other hand, any indecomposable extension of $M_{1}$ by $M_{0}$ is isomorphic to $M$.

Consider now the $R$-module $\left|U_{6}\right|=\left\{|\varepsilon| \mid \varepsilon \in U_{6}\right\}$. It is not hard to see that

$$
\begin{aligned}
& \left|U_{2} U_{3}\right|=\left\langle\mu,|\tau|,\left|\tau^{\prime}\right|\right\rangle=\left\{x \in\left|U_{6}\right| \mid x^{(1+\sigma)\left(1+\sigma+\sigma^{2}\right)}=1\right\} \cong M_{0}, \\
& \left|U_{6}\right| /\left|U_{2} U_{3}\right| \cong\left|U_{R}\right| \cong M_{1} .
\end{aligned}
$$

Therefore, $\left|U_{6}\right|$ is also an extension of $M_{1}$ by $M_{0}$.

THEOREM 1. The structure of the $R$-module $\left|U_{6}\right|$, in particular its decomposition into indecomposable direct summands, can be described as follows:

$$
\left|U_{6}\right| \cong \begin{cases}R /\left(1+\sigma+\cdots+\sigma^{5}\right) R & \text { if } Q_{K}=12, \\ R /\left(1+\sigma+\sigma^{2}\right) R \oplus R /\left(1+\sigma^{3}\right) R & \text { if } Q_{K}=3, \\ R /(1+\sigma) R \oplus R /\left(1+\sigma^{2}+\sigma^{4}\right) R & \text { if } Q_{K}=4, \\ R /(1+\sigma) R \oplus R /\left(1+\sigma+\sigma^{2}\right) R \oplus R /\left(1-\sigma+\sigma^{2}\right) R & \text { if } Q_{K}=1 .\end{cases}
$$

Proof. As stated above, we consider $\left|U_{6}\right|$ as an extension of $\left|U_{6}\right| /\left|U_{2} U_{3}\right|$ by $\left|U_{2} U_{3}\right|$. Let $\Lambda$ be a cocycle corresponding to this extension and choose

$$
\lambda \in \operatorname{Hom}_{\mathbf{z}}\left(\left|U_{6}\right| /\left|U_{2} U_{3}\right|,\left|U_{6}\right|\right)
$$


to satisfy $p \circ \lambda=1$ and (4.1), mutatis mutandis. From the equations (4.2) we get

$$
\begin{aligned}
f_{\Lambda}=0 & \Leftrightarrow \lambda(x)^{1+\sigma^{2}+\sigma^{4}} \in\left|U_{2} U_{3}\right|^{3} \quad \text { for each } x \in\left|U_{6}\right| /\left|U_{2} U_{3}\right| \\
& \Leftrightarrow N_{6 / 2}\left(U_{6}\right) \neq U_{2}, \\
g_{\Lambda}=0 & \Leftrightarrow \lambda(x)^{1+\sigma^{3}} \in\left|U_{2} U_{3}\right|^{2} \quad \text { for each } x \in\left|U_{6}\right| /\left|U_{2} U_{3}\right| \\
& \Leftrightarrow\langle-1\rangle N_{6 / 3}\left(U_{6}\right) \neq U_{3} .
\end{aligned}
$$

In the case $Q_{K}=12$, we thus find immediately that $\left|U_{6}\right| \cong M$. Consider now, e.g., the case $Q_{K}=4$, i.e.,

$$
f_{\Lambda}=0, \quad g_{\Lambda} \neq 0 .
$$

Then $\left|U_{6}\right|$ is isomorphic to a direct sum of $R /(1+\sigma) R$ and an indecomposable extension of $R /\left(1-\sigma+\sigma^{2}\right) R$ by $R /\left(1+\sigma+\sigma^{2}\right) R$. The latter is necessarily isomorphic to $R /\left(1+\sigma^{2}+\sigma^{4}\right) R$. In the other two cases one can argue similarly.

A unit $\varepsilon$ of the ring $\mathcal{O}_{6}$ is called a Minkowski unit iff $\left|U_{6}\right|$ is a cyclic $R$-module and $|\varepsilon|$ is an $R$-generator of $\left|U_{6}\right|$. We thus see that a Minkowski unit exists if and only if $Q_{K}=12$.

5. Unit Norms. Put

$$
\kappa= \begin{cases}0 & \text { if } \chi_{6} \text { is decomposable } \\ 1 & \text { if } \chi_{6} \text { is nondecomposable }\end{cases}
$$

This notation enables one to state the results below in a compact form covering both the decomposable case and the nondecomposable ones.

The relative norms of the unit $\xi_{A}$ to the fields $K_{2}$ and $K_{3}$ are important characteristics, and the numbers $u, v, w$ defined by

$$
N_{6 / 3}\left(\xi_{A}\right)= \pm \tau^{u} \tau^{\prime \prime}, \quad N_{6 / 2}\left(\xi_{A}\right)= \pm \mu^{u}
$$

are listed in the tables. We shall now derive rather precise results about these numbers. In the subsequent discussion $n=2,3$ or 6 .

Let $\xi_{n}$ be "die Basiszahl der Kreiseinheit" in Leopoldt's terminology. From [11, p. $37,(3)]$, we have

$$
\xi_{n}^{2}= \pm N_{C\left(f_{n}\right) / K_{n}}\left(1-\zeta_{f_{n}}\right)
$$

If $\eta_{n}$ denotes the cyclotomic unit of $K_{n}$, then

$$
\eta_{n}^{2}=\xi_{n}^{2(1-\sigma)} \text {. }
$$

The formula has been written with an extra factor 2 in the exponent in order to ensure that the number upon which $1-\sigma$ operates belongs to $K_{n}$. We also denote briefly $\xi=\xi_{6}, \eta=\eta_{6}$. Note that $\xi_{A}=\xi$ if $\chi_{6}$ is decomposable, and $\xi_{A}=\eta$ otherwise.

From $\left[9\right.$, p. $\left.67,\left(6^{*}\right)\right]$, we have

$$
\eta_{2}= \pm \mu^{-h_{2}}
$$

and from [10, p. 40, Satz 9],

$$
h_{3}=\left[U_{3}: Y_{3}\right]=\left[\left\langle-1, \tau, \tau^{\prime}\right\rangle:\left\langle-1, \eta_{3}, \eta_{3}^{\prime}\right\rangle\right] \text {. }
$$


The value of $w$ is completely determined by the following result.

TheOrem 2. Denote $f_{6}=3^{\lambda} p_{1} \cdots p_{\nu} f_{2}$ where $\lambda \in\{0,1,2\}, \nu \geqslant 0$, and the $p_{i}$ 's are distinct primes $\equiv 1 \bmod 6$.

(i) If there exists an index $i \in\{1,2, \ldots, \nu\}$ such that $\left(f_{2} / p_{i}\right)=1$ or if $\lambda=2$ and $f_{2} \equiv 1 \bmod 3$, then $w=0$.

(ii) Otherwise,

$$
w=-2^{\nu+\kappa+\max \{1, \lambda\}-2} h_{2} .
$$

Proof. For any prime $p$ not dividing $f_{n}$, let $\sigma(n, p)$ denote the restriction to the field $K_{n}$ of the automorphism of $C\left(f_{n}\right)$ induced by $\zeta_{f_{n}} \mapsto \zeta_{f_{n}}^{p}$. From [11, p. 38, (5)], we have

$$
N_{6 / n}\left(\xi^{2}\right)= \pm \xi_{n}^{2 W_{n}}
$$

where

$$
W_{n}=\prod_{p \mid f_{6}, p \nmid f_{n}}\left(1-\sigma(n, p)^{-1}\right) .
$$

Here we take $n=2$ in (5.6) and (5.7). Since $\sigma(2, p)=\left(f_{2} / p\right)$ the assertion (i) follows immediately. Suppose therefore that $\left(f_{2} / p_{i}\right)=-1$ for each $i$ and consider first the case $\lambda \neq 2$. Then $p=3$ does not appear in (5.7). If $\chi_{6}$ is decomposable, we obtain from (5.1), (5.6), (5.3), (5.4),

$$
\mu^{4 w}=N_{6 / 2}\left(\xi^{4}\right)=\xi_{2}^{4 W_{2}}=\xi_{2}^{2(1-\sigma) 2^{\nu}}=\eta_{2}^{2^{v+1}}=\mu^{-2^{v+1} h_{2}} .
$$

Hence $w=-2^{\nu-1} h_{2}$ as asserted. If $\chi_{6}$ is nondecomposable, we obtain similarly

$$
\mu^{2 w}=N_{6 / 2}\left(\eta^{2}\right)=N_{6 / 2}\left(\xi^{2(1-\sigma)}\right)=\xi_{2}^{2(1-\sigma) W_{2}}=\eta_{2}^{2 W_{2}}=\eta_{2}^{2^{\nu+1}}=\mu^{-2^{\nu+1} h_{2}},
$$

whence $w=-2^{\nu} h_{2}$.

If $\lambda=2$ and $f_{2} \equiv 2 \bmod 3$ we have the same situation as above with the prime 3 added to the set $\left\{p_{1}, \ldots, p_{\nu}\right\}$.

A corresponding result for the pair $(u, v)$ is

THEOREM 3. Denote $f_{6}=p_{1}^{\lambda} p_{2} \cdots p_{\nu} f_{3}$, where the $p_{i}$ 's are distinct primes, and $\lambda=1$ if $\nu>0$ and $f_{6}$ is odd, while $p_{1}=2$ and $\lambda \in\{2,3\}$ if $f_{6}$ is even.

(i) If there exists an index $i \in\{1,2, \ldots, \nu\}$ such that $\chi_{3}\left(p_{i}\right)=1$, then $(u, v)=(0,0)$.

(ii) Otherwise $u^{2}-u v+v^{2}=3^{v+\kappa-1} h_{3}$.

Proof. If $\gamma \neq \pm 1$ is a unit in $U_{3}$, let $j(\gamma)$ denote the index $\left[U_{3}:\left\langle-1, \gamma, \gamma^{\prime}\right\rangle\right]$. Using this notation (5.5) takes the form $h_{3}=j\left(\eta_{3}\right)$. A simple computation gives

$$
j\left( \pm \gamma^{x+y \sigma}\right)=\left(x^{2}-x y+y^{2}\right) j(\gamma) \text { for }(x, y) \in \mathbf{Z}^{2} \backslash\{(0,0)\} .
$$

Take $n=3$ in (5.6) and (5.7) and put $P=p_{1}^{\lambda} p_{2} \cdots p_{\nu}=f_{6} / f_{3}$. Define $\delta(x)$ for any integer $x$ prime to $f_{3}$ by

$$
\delta(x)=\sigma^{2 k} \text { if } \chi_{3}(x)=\rho^{k} \quad(\rho=\exp (2 \pi i / 3), k \in\{0,1,2\}) .
$$

According to the agreement in [13, p. 9 and p. 12], the choice of $\sigma$ and $\chi_{3}$ was made so that for $\left(x, f_{3}\right)=1$ the automorphism of $C\left(f_{3}\right)$ induced by $\zeta_{f_{3}} \mapsto \zeta_{f_{3}}^{x}$ and $\sigma$ have the same restriction to the field $K_{3}$ if and only if $\chi_{3}(x)=\rho$. Therefore, the element $W_{3}$ in (5.7) is

$$
W_{3}=\prod_{p \mid P}(1-\delta(p))
$$


If $\nu>0$ and $\chi_{3}\left(p_{i}\right)=1$ for some $i$ then $W_{3}=0$ and the assertion (i) follows. Suppose the contrary. If $\chi_{6}$ is decomposable, it follows from (5.1) and (5.6) that $\tau^{2 u+2 v \sigma}= \pm \xi_{3}^{2 W_{3}}$, and thus (5.8), (5.3), (5.5) imply

$$
4\left(u^{2}-u v+v^{2}\right)=3^{\nu-1} j\left(\xi_{3}^{2(1-\sigma)}\right)=3^{\nu-1} j\left(\eta_{3}^{2}\right)=4 \cdot 3^{\nu-1} h_{3},
$$

as asserted. Suppose, therefore, that $\chi_{6}$ is nondecomposable. In this case $\tau^{2 u+2 v \sigma}=$ $\xi_{3}^{2(1-\sigma) W_{3}}$ and we have the same computation with $3^{\nu-1}$ replaced by $3^{\nu}$.

If $K_{6}^{\prime}$ and $K_{6}^{\prime \prime}$ have the same conductor $f_{6}$ and the same cubic subfield $K_{3}$, and if the corresponding characters $\chi_{6}^{\prime}, \chi_{6}^{\prime \prime}$ are both decomposable, it is obvious by (5.2), (5.3) (for $n=6$ ) and by (5.1), that the pair $(u, v)$ is the same for both fields $K_{6}^{\prime}, K_{6}^{\prime \prime}$. The same is trivially true if $\chi_{6}^{\prime}, \chi_{6}^{\prime \prime}$ are both nondecomposable, because in that case, $K_{6}^{\prime}=K_{6}^{\prime \prime}$ as is easily seen.

6. Ideal classes of subfields becoming principal in $K_{6}$. Although the class number $h_{6}$ is divisible by the product $h_{2} h_{3}$, it is not always true that the ideal classes of the subfields are mapped injectively under the natural mapping induced by the inclusion. This phenomenon of capitulation has been studied by M.-N. Gras [6], [7] in the case of a real cyclic field of degree four. Here we shall carry out a similar investigation in the sextic case. We remind the reader of the notations introduced in Section 2. In particular, we shall assume that $N_{6 / 2}\left(\xi_{R}\right)=1$. We note that $N_{6 / 3}(\varepsilon)=1$ for each $\varepsilon \in U_{R}[13$, p. 14, (22)]. In the following discussion $n=2$ or 3 , indicating that the subfield under consideration is $K_{n}$.

Suppose that $\mathrm{Cl}_{n}(\mathfrak{c}) \in \mathscr{C}_{6 / n}$ and that $\mathrm{cO}_{6}=\gamma \mathcal{O}_{6}$ for some $\gamma \in K_{6} \backslash\{0\}$. It is obvious that the assignment $g_{n}\left(\mathrm{Cl}_{n}(\mathrm{c})\right)=\gamma^{1-\sigma^{n}} U_{6}^{1-\sigma^{n}}$ gives a well-defined homomorphism $g_{n}: \mathscr{C}_{6 / n} \rightarrow U_{R n} / U_{6}^{1-\sigma^{n}}$. Further, it is easy to see that $g_{n}$ is injective. Suppose, namely, that $\gamma^{1-\sigma^{n}}=\varepsilon^{1-\sigma^{n}}$, where $\varepsilon \in U_{6}$. We have $\gamma / \varepsilon=(\gamma / \varepsilon)^{\sigma^{n}}$ so that $\gamma / \varepsilon \in K_{n}$. Since $c \mathcal{O}_{6}=(\gamma / \varepsilon) \mathcal{O}_{6}$ it follows that $c=(\gamma / \varepsilon) \mathcal{O}_{n}$ belongs to the principal class.

Suppose now that $\varepsilon U_{6}^{1-\sigma^{n}}$ is an arbitrary element of $U_{R n} / U_{6}^{1-\sigma^{n}}$. By Theorem 90 of Hilbert there is a $\gamma \in \mathcal{O}_{6} \backslash\{0\}$ such that $\varepsilon=\gamma^{1-\sigma^{n}}$. Since the automorphism $\sigma^{n}$ leaves the ideal $\gamma \mathcal{O}_{6}$ fixed, we have

$$
\gamma \mathcal{O}_{6}=\mathfrak{c O}_{6} \times \mathfrak{p}_{1}^{\nu_{1}} \times \cdots \times \mathfrak{p}_{h}^{\nu_{h}}
$$

where $c$ is an ideal of $\mathcal{O}_{n}$ and the $\mathfrak{p}_{i}$ 's are distinct prime ideals of $\mathcal{O}_{6}$ which are ramified in $K_{6} / K_{n}$. We may assume that, for each $i, \mathfrak{p}_{i}+\mathfrak{c}$ so that $\nu_{i}=\nu_{\mathfrak{p}_{i}}(\gamma)$.

Lemma 1. We have $\varepsilon U_{6}^{1-\sigma^{n}} \in \operatorname{Im}\left(g_{n}\right)$ if and only if $n \nu_{i} \equiv 0 \bmod 6$ for $i=1$, $2, \ldots, h$.

Proof. Both conditions are clearly equivalent to the fact that $\gamma \mathcal{O}_{6}=\mathfrak{c}_{0} \mathcal{O}_{6}$ for some ideal $\mathrm{c}_{0}$ of $\mathcal{O}_{n}$.

The number $\omega$ was defined as $\omega=\tau^{-1}+\xi_{R} \tau^{\prime \prime-1}+\xi_{R}^{\prime} \tau^{\prime-1}$. We have $\omega^{\sigma^{2}}=\xi_{R}^{-1} \omega$ so that $\omega$ satisfies Hilbert 90 in the extension $K_{6} / K_{2}$ for $\xi_{R}$ provided that $\omega \neq 0$. Here we shall investigate the possibility $\omega=0$ which incidentally leads to a parametric family of relative units in certain fields $K_{6}$. More generally, we shall prove the following result. 
THEOREM 4. Let $\delta \neq 1$ be a norm-positive unit in a cyclic cubic field $K_{3}$. Put $M=S_{3 / 1}\left(\delta^{-4}-2 \delta^{2}\right)$. Then, $M \geqslant 0$ with equality only when $\operatorname{Irr}(\delta, \mathbf{Q})=x^{3}+3 x^{2}-$ 1. Suppose that $M$ is not a square in $\mathbf{Z}$ and write $M=c^{2} m$, where $c \in \mathbf{Z}$ and $m$ is $a$ square-free positive integer.

The number

$$
\psi=\left(-\delta^{-2}+\delta^{\prime-2}-\delta^{\prime \prime-2}+c \sqrt{m}\right) /\left(2 \delta^{\prime}\right)
$$

is a unit in the field $K_{3}(\sqrt{m})$, such that

$$
N_{6 / 2}(\psi)=N_{6 / 3}(\psi)=1, \quad \delta^{-1}+\psi \delta^{\prime \prime-1}+\psi^{\prime} \delta^{\prime-1}=0 .
$$

Conversely, let $K_{6}$ be any real cyclic sextic field containing $K_{3}$ and let $\psi$ be a unit in $K_{6}$ satisfying (6.2). Then, $K_{6}=K_{3}(\sqrt{m})$ and $\psi$ is of the form (6.1) where both signs for $c$ are permitted.

Proof. We shall first prove the assertion concerning $M$. Let $\operatorname{Irr}(\delta, \mathbf{Q})=x^{3}-s x^{2}$ $+q x-1$. Then, $M=q^{4}-4 s q^{2}+8 q$. Since the discriminant $\operatorname{or} \operatorname{Ir}(\delta, \mathbf{Q})$ is a square $\geqslant 49$ in $\mathbf{Z}$, we have

$$
s^{2} q^{2}-4 q^{3}-4 s^{3}-27+18 s q=t^{2}
$$

for some integer $t \geqslant 7$. Consider first the possibility $q=0$. Then, $-4 s^{3}-27=t^{2}$. Using a trick due to Fueter we can write this as a Fermat equation

$$
(2 s)^{3}+(3+t / 3)^{3}+(3-t / 3)^{3}=0 .
$$

The only solution is thus $t=9, s=-3$, which is the exceptional case mentioned in the theorem. Suppose now that $q \neq 0$. We contend that $M>0$. Write $N=M /\left(4 q^{2}\right)$ so that

$$
s=q^{2} / 4+2 / q-N
$$

Substituting this in (6.3), we obtain

$$
4 N^{3}-\left(2 q^{2}+24 / q\right) N^{2}+\left(q^{4} / 4-10 q+48 / q^{2}\right) N+1-32 / q^{3}=t^{2} .
$$

For $q \geqslant 4$ or $q=1$ or $q \leqslant-3$ the expressions in parentheses in (6.4) are positive and therefore $t \geqslant 7$ implies $N>0$. For the remaining values $q=-2,-1,2,3$ it is easy to verify by direct computation that $t \geqslant 7$ is possible only if $N>0$. This proves the first assertion.

In what follows, we exclude the exceptional case and we assume further that $M$ is not a square in $\mathbf{Z}$. We write $M=c^{2} m$ as indicated and define $\psi$ by (6.1). We have

$$
\begin{aligned}
4 \delta^{\prime 2} \psi \psi^{\prime \prime \prime} & =S_{3 / 1}\left(\delta^{-4}\right)-2 \delta^{2}+2 \delta^{\prime 2}-2 \delta^{\prime \prime 2}-M \\
& =2 S_{3 / 1}\left(\delta^{2}\right)-2 \delta^{2}+2 \delta^{\prime 2}-2 \delta^{\prime \prime 2}=4 \delta^{\prime 2},
\end{aligned}
$$

whence $N_{6 / 3}(\psi)=\psi \psi^{\prime \prime \prime}=1$. As $\psi+\psi^{\prime \prime \prime} \in \mathcal{O}_{3}, \psi$ is an algebraic integer and, therefore, a unit in $K_{6}=K_{3}(\sqrt{m})$. Put

$$
h(x)=\operatorname{Irr}\left(\delta^{-2}, \mathbf{Q}\right)=x^{3}-\left(q^{2}-2 s\right) x^{2}+\left(s^{2}-2 q\right) x-1 .
$$

We have

$$
\begin{aligned}
-N_{6 / 2}(\psi) & =-N_{6 / 2}\left(\delta^{\prime} \psi\right)=N_{6 / 2}\left(\left(q^{2}-2 s-c \sqrt{m}\right) / 2-\delta^{\prime-2}\right) \\
& =h\left(\left(q^{2}-2 s-c \sqrt{m}\right) / 2\right)=-1
\end{aligned}
$$


and, finally,

$$
\begin{aligned}
\delta^{-1}+\psi \delta^{\prime \prime-1}+\psi^{\prime} \delta^{\prime-1}= & \delta^{-1}+\frac{1}{2} \delta\left(-\delta^{-2}+\delta^{\prime-2}-\delta^{\prime \prime-2}+c \sqrt{m}\right) \\
& +\frac{1}{2} \delta\left(-\delta^{\prime-2}+\delta^{\prime \prime-2}-\delta^{-2}-c \sqrt{m}\right)=0
\end{aligned}
$$

Hence (6.2) is satisfied.

Now, suppose, conversely, that $\psi$ is a unit satisfying (6.2) in some real cyclic sextic field $K_{6}$ containing $K_{3}$. Combining the last condition (6.2) and the one obtained from it by applying the automorphism $\sigma^{3}$, we find

$$
\delta^{\prime}\left(\psi-\psi^{-1}\right)=-\delta^{\prime \prime}\left(\psi^{\prime}-\psi^{\prime-1}\right)
$$

Denote $c_{*}=\delta^{\prime}\left(\psi-\psi^{-1}\right) / \sqrt{m}$. It follows from (6.5) that $\sigma$ leaves $c_{*}$ fixed so that $c_{*} \in \mathbf{Q}$. Further,

$$
\psi=\left(\gamma+c_{*} \sqrt{m}\right) /\left(2 \delta^{\prime}\right),
$$

where $\gamma \in K_{3}$, and the last condition (6.2) also gives

$$
\delta^{-1}+\gamma \delta^{\prime \prime-1}+\gamma^{\prime} \delta^{\prime-1}=0 .
$$

Applying the automorphism $\sigma$ twice to (6.6), we get a system of three equations from which we can solve $\gamma, \gamma^{\prime}, \gamma^{\prime \prime}$. In that way we obtain $\gamma=\left(-\delta^{-2}+\delta^{\prime-2}-\delta^{\prime \prime-2}\right) /\left(2 \delta^{\prime}\right)$ and the condition $N_{6 / 3}(\psi)=1$ gives $c_{*}^{2} m=M=c^{2} m$, i.e., $c_{*}= \pm c$.

Consider, in particular, the case $q=-s-3$, i.e., $\operatorname{Irr}(\delta, \mathbf{Q})=x^{3}-s x^{2}-(s+3) x$ - 1. This polynomial is clearly irreducible in $\mathbf{Q}[x]$ for any integer $s$. The discriminant of the polynomial is $\left(s^{2}+3 s+9\right)^{2}$ so that $\mathbf{Q}(\delta) / \mathbf{Q}$ is cyclic. In this case, we have

$$
M=q^{4}-4 s q^{2}+8 q=s^{4}+8 s^{3}+30 s^{2}+64 s+57 .
$$

E.g., for $s \equiv 1 \bmod 8$ it is easily seen that $M \equiv 32 \bmod 64$ so that, for these values of $s, M$ is not a square in $\mathbf{Z}$. Further, it is not hard to see that $s^{2}+3 s+9$ is square-free for infinitely many $s \equiv 1 \bmod 8$, and, therefore, there are infinitely many different fields $K_{3}$. In the corresponding family of sextic fields we have an explicit system $\left\{\delta, \delta^{\prime}, \psi, \psi^{\prime}\right\}$ of four independent units. Unfortunately, we have not been able to find an expression for a missing fifth unit in terms of the parameter $s$.

It is of interest to observe that if a unit $\psi$ satisfying (6.2) exists in $K_{3}(\sqrt{m})$ then $\psi \delta \delta^{\prime \prime-1}$ is an exceptional unit in the terminology of Nagell [14].

7. Classes of $K_{2}$ in $K_{6}$. In this section we shall assume that $\omega=\tau^{-1}+\xi_{R} \tau^{\prime \prime-1}+$ $\xi_{R}^{\prime} \tau^{\prime-1} \neq 0$. Namely, by Theorem 4 it is obvious that if the original $\omega$ vanishes, we obtain a nonzero $\omega$ on replacing $\xi_{R}$ by $\xi_{R}^{\prime}$. We denote $\xi_{R}=\alpha+\beta \sqrt{m}$, where $\alpha$, $\beta \in K_{3}$. It follows easily from $N_{6 / 2}\left(\xi_{R}\right)=N_{6 / 3}\left(\xi_{R}\right)=1$, that

$$
\begin{gathered}
\xi_{R}=\omega^{1-\sigma^{2}}, \\
\omega^{1+\sigma^{3}}=S_{3 / 1}\left(\tau^{-2}+2 \alpha \tau^{\prime}\right) \in \mathbf{Z} .
\end{gathered}
$$

In the verification it is useful to note that, in particular, $\xi_{R} \xi_{R}^{\prime \prime}=\xi_{R}^{\prime}$.

From [8, Ia, p. 92, Satz 12] we have

$$
\left[U_{R 2}: U_{6}^{1-\sigma^{2}}\right]=3^{2-q},
$$

where

$$
3^{q}=\left[N_{6 / 2}\left(U_{6}\right): U_{2}^{3}\right]= \begin{cases}3 & \text { if } N_{6 / 2}\left(U_{6}\right)=U_{2} \\ 1 & \text { if } N_{6 / 2}\left(U_{6}\right) \neq U_{2}\end{cases}
$$


LEMMA 2. A system of coset representatives of $U_{R 2}$ with respect to the subgroup $U_{6}^{1-\sigma^{2}}$ is

$$
\begin{array}{ll}
\left\{\tau^{i} \mid i=0,1,2\right\} & \text { if } N_{6 / 2}\left(U_{6}\right)=U_{2} \\
\left\{\tau^{i} \xi_{R}^{j} \mid i, j=0,1,2\right\} & \text { if } N_{6 / 2}\left(U_{6}\right) \neq U_{2}
\end{array}
$$

Proof. If $\tau \in U_{6}^{1-\sigma^{2}}$ there exists an $\varepsilon \in U_{6}$ such that $\tau=\varepsilon^{1-\sigma^{2}}$. Taking norms, we have $\tau^{2}=\varepsilon^{\left(1+\sigma^{3}\right)\left(1-\sigma^{2}\right)}$, so that (5.8) implies

$$
4=j\left(\tau^{2}\right)=3 j\left(\varepsilon^{1+\sigma^{3}}\right),
$$

which is impossible. In the case $N_{6 / 2}\left(U_{6}\right)=U_{2}$ the assertion therefore follows from (7.3) and (7.4).

Suppose now that $N_{6 / 2}\left(U_{6}\right) \neq U_{2}$ and that $\tau^{i} \xi_{R}=\varepsilon^{1-\sigma^{2}}$ for some $i \in\{0,1,2\}$ and some $\varepsilon \in U_{6}$. On multiplying $\varepsilon$ by $\pm \mu^{k}$ for a suitable $k$, we may assume that $N_{6 / 2}(\varepsilon)=1$. Taking norms, we have $\tau^{2 i}=\varepsilon^{\left(1+\sigma^{3}\right)\left(1-\sigma^{2}\right)}$ which, for $i \neq 0$, leads to the same contradiction as above. Thus, $i=0, \xi_{R}=\varepsilon^{1-\sigma^{2}}$ and $\varepsilon^{\left(1+\sigma^{3}\right)\left(1-\sigma^{2}\right)}=1$. It follows that $\varepsilon^{1+\sigma^{3}} \in K_{3} \cap K_{2}=\mathbf{Q}$, i.e. $\varepsilon^{1+\sigma^{3}}= \pm 1$. Therefore, $\varepsilon \in U_{R}$. However, from $\xi_{R}=\varepsilon^{1-\sigma^{2}}$ it is easy to infer that

$$
\left[\left\langle-1, \varepsilon, \varepsilon^{\prime}\right\rangle:\left\langle-1, \xi_{R}, \xi_{R}^{\prime}\right\rangle\right]=3,
$$

which is impossible. The assertion follows again from (7.3) and (7.4)

THEOREM 5. If $i \neq \equiv 0 \bmod 3$ we have $\tau^{i} \xi_{R}^{j} U_{6}^{1-\sigma^{2}} \notin \operatorname{Im}\left(g_{2}\right)$ for each $j$. In particular, $\mathscr{C}_{6 / 2}=1$ if $N_{6 / 2}\left(U_{6}\right)=U_{2}$.

Proof. From Hilbert 90 we have $\tau^{i} \xi_{R}^{j}=\gamma^{1-\sigma^{2}}$ for some $\gamma \in \mathcal{O}_{6} \backslash\{0\}$. If $\tau^{i} \xi_{R}^{j} U_{6}^{1-\sigma^{2}}$ $\in \operatorname{Im}\left(g_{2}\right)$ there exists an ideal $c$ of $\mathcal{O}_{2}$ such that $\mathrm{cO}_{6}=\gamma \mathcal{O}_{6}$. Applying the automorphism $\sigma^{3}$, we have $\gamma^{1+\sigma^{3} \mathcal{O}_{6}}=c^{1+\sigma^{3} \mathcal{O}_{6}}=c \mathcal{O}_{6}$, say, for some $c \in \mathbf{Z}$. Therefore, $\gamma^{1+\sigma^{3}}=$ $c \varepsilon$, where $\varepsilon \in U_{3}$. We would have

$$
\tau^{2 i}=\left(\tau^{i} \xi_{R}^{j}\right)^{1+\sigma^{3}}=\varepsilon^{1-\sigma^{2}},
$$

which leads to the same contradiction as in the proof of Lemma 2. The rest of the assertion is clear because $g_{2}$ is injective.

From now on, in this section we shall assume that $N_{6 / 2}\left(U_{6}\right) \neq U_{2}$. From Lemma 2 and Theorem 5 we see that $\operatorname{Im}\left(g_{2}\right)$ is either 1 or $\left\langle\xi_{R} U_{6}^{1-\sigma^{2}}\right\rangle$, i.e., \#数/2 $=1$ or 3 . Our numerical results indicate that the latter alternative may always hold if $N_{6 / 2}\left(U_{6}\right) \neq U_{2}$ and $h_{2} \equiv 0 \bmod 3$.

From Lemma 1 and (7.1) we have, immediately,

LEMMA 3. If $N_{6 / 2}\left(U_{6}\right) \neq U_{2}$ and $h_{2} \equiv 0 \bmod 3$, then $\# \mathscr{C}_{6 / 2}=3$ if and only if $\nu_{\mathfrak{p}}(\omega) \equiv 0 \bmod 3$ for every prime ideal $\mathfrak{p}$ of $\mathcal{O}_{6}$ which is ramified in the extension $K_{6} / K_{2}$.

The following theorem gives a practical criterion by means of which we have been able to establish the truth of the above conjectural fact for every field $K_{6}$ up to $f_{6}<4000$. 
THEOREM 6. Let $\xi_{R}=\alpha+\beta \sqrt{m}$, where $\alpha, \beta \in K_{3}$. Suppose that $N_{6 / 2}\left(\xi_{R}\right)=1$ and that $\omega=\tau^{-1}+\xi_{R} \tau^{\prime \prime-1}+\xi_{R}^{\prime} \tau^{\prime-1} \neq 0$. Let $\mathfrak{p}$ be a prime ideal of $\mathcal{O}_{6}$ which is ramified in the extension $K_{6} / K_{2}$. Write $\mathfrak{p} \cap \mathbf{Z}=p \mathbf{Z}$, where $p$ is a prime, and $\omega=\varphi+\psi \sqrt{m}$, where $\varphi, \psi \in K_{3}$.

(i) If $p$ is ramified or inert in $K_{2} / \mathbf{Q}$, then $\nu_{p}(\omega) \equiv 0 \bmod 3$.

(ii) If $p$ splits in $K_{2} / \mathbf{Q}$ and $p \neq 3$, then the following conditions are equivalent:

$$
\mathfrak{p} \mid \omega \Leftrightarrow \alpha \equiv-\frac{1}{2} \bmod \mathfrak{p} \Leftrightarrow \nu_{\mathfrak{p}}(\omega) \neq \equiv 0 \bmod 3 .
$$

(iii) Suppose that $p=3$ splits in $K_{2} / \mathbf{Q}$. Let $3^{k} \| S_{3 / 1}\left(\tau^{-1}\right)(\neq 0 ; c f .[13, p .64])$ and $l=\nu_{\mathrm{p}}(\beta)$.

If $3 k \neq 2 l$, then

$$
\nu_{\mathfrak{p}}(\omega) \not \equiv 0 \bmod 3 \Leftrightarrow l=1
$$

If $3 k=2 l$, then

$$
\nu_{\mathfrak{p}}(\omega) \not \equiv 0 \bmod 3 \Leftrightarrow \nu_{p}(\varphi)=\nu_{\mathfrak{p}}(\psi) \text { with common value } \not \equiv 0 \bmod 3 \text {. }
$$

Proof. (i) We have $p \mathcal{O}_{6}=\mathfrak{p}^{3}$ or $\mathfrak{p}^{6}$. Since $\omega^{1+\sigma^{3}} \in \mathbf{Z}$ by (7.2), $\nu_{\mathfrak{p}}\left(\omega^{1+\sigma^{3}}\right) \equiv 0 \bmod 3$. Since $\sigma^{3}$ leaves $\mathfrak{p}$ fixed, the result follows.

(ii) Put $A=\omega^{1+\sigma^{3}}$. We have

$$
\varphi=\tau^{-1}+\alpha \tau^{\prime \prime-1}+\alpha^{\prime} \tau^{\prime-1}, \quad \psi=\beta \tau^{\prime \prime-1}-\beta^{\prime} \tau^{\prime-1}
$$

and from (7.5) and (7.2),

$$
\varphi^{2}-m \psi^{2}=A=S_{3 / 1}\left(\varphi \tau^{-1}\right) .
$$

Since $p+2 f_{2}$, the number $\beta$ is $p$-integral so that (7.5) implies

$$
\varphi \equiv(1+2 \alpha) \tau^{-1} \bmod \mathfrak{p}, \quad \psi \equiv 0 \bmod \mathfrak{p} .
$$

It is clear by (7.7) that $\mathfrak{p} \mid \omega$ iff $\alpha \equiv-\frac{1}{2} \bmod \mathfrak{p}$ and that these conditions follow from $\nu_{\mathfrak{p}}(\omega) \neq \equiv 0 \bmod 3$. We therefore assume that $\mathfrak{p} \mid \omega$ and contend that $\nu_{\mathfrak{p}}(\omega) \neq \equiv 0 \bmod 3$.

We note that the following result holds:

$$
\text { If } \gamma \in K_{3} \text { is } \mathfrak{p} \text {-integral, then } \mathfrak{p} \mid \gamma \text { iff } p \mid S_{3 / 1}(\gamma) \text {. }
$$

This follows immediately from $S_{3 / 1}(\gamma) \equiv 3 \gamma \bmod \mathfrak{p}$ and $\mathfrak{p}+3$.

We shall show first that $\nu_{\mathfrak{p}}(\psi) \neq \equiv \bmod 3$. Suppose, namely, that $\nu_{\mathfrak{p}}(\psi)=3 n$. Then $\tau^{-1} \psi p^{-n}$ is $p$-integral and not divisible by $\mathfrak{p}$. On the other hand, by (7.5),

$$
S_{3 / 1}\left(\tau^{-1} \psi p^{-n}\right)=p^{-n}\left(S_{3 / 1}\left(\tau^{\prime} \beta\right)-S_{3 / 1}\left(\tau^{\prime \prime} \beta^{\prime}\right)\right)=0,
$$

which contradicts (7.8).

We shall show next that $\nu_{p}(\varphi) \neq 0 \bmod 3$. Suppose, on the contrary, that $\nu_{p}(\varphi)=$ $3 n$. From (7.6), we have $\nu_{\mathfrak{p}}(A)=\nu_{\mathfrak{p}}\left(\varphi^{2}\right)=6 n$ and $S_{3 / 1}\left(\varphi \tau^{-1}\right) \equiv 0 \bmod p^{2 n}$. On the other hand, $\varphi \tau^{-1} p^{-n}$ is $p$-integral and prime to $p$ so that (7.8) implies $p^{-n} S_{3 / 1}\left(\varphi \tau^{-1}\right)$ $\not \equiv 0 \bmod p$, a contradiction.

From (7.6) we now have $\nu_{p}(\varphi)=\nu_{p}(\psi)=n$, say, where $3+n$. Since $\omega=\varphi+\psi \sqrt{m}$ and $\omega+\omega^{\prime \prime \prime}=2 \varphi$, it follows that $\mathfrak{p}^{n} \|\left(\omega, \omega^{\prime \prime \prime}\right)$. Since $\nu_{\mathfrak{p}}(\omega)+\nu_{\mathfrak{p}}\left(\omega^{\prime \prime \prime}\right)=\nu_{\mathfrak{p}}(A) \equiv 0$ $\bmod 3$, it is clear that $\nu_{p}(\omega) \neq 0 \bmod 3$.

(iii) The conditions (7.7) are plainly also true in the case $p=3$. From $A=$ $S_{3 / 1}\left(\varphi \tau^{-1}\right) \equiv 0 \bmod 3$ we have $\mathfrak{p} \mid \varphi$ whence by (7.7), $\alpha \equiv 1 \bmod \mathfrak{p}$. From the equation $(\alpha+1)(\alpha-1)=m \beta^{2}$ we find that $\mathfrak{p} \mid \beta$, i.e., $l>0$ and then $\nu_{\mathfrak{p}}(\alpha-1)=$ $2 l$. 
Case $1.3 k<2 l$. Since $k>0$ we have $l>1$ and we thus contend that $\nu_{\mathfrak{p}}(\omega) \equiv 0$ mod 3. Writing

$$
\varphi=S_{3 / 1}\left(\tau^{-1}\right)+(\alpha-1) \tau^{\prime \prime-1}+\left(\alpha^{\prime}-1\right) \tau^{\prime-1}
$$

we find that $\nu_{\mathfrak{p}}(\varphi)=3 k$. It is clear by (7.6) that if $\nu_{\mathfrak{p}}(\psi) \neq \equiv \bmod 3$, then necessarily $\nu_{p}(\psi)>3 k$. Using the same argument as at the end of (ii), we obtain first $\nu_{\mathrm{p}}\left(\left(\omega, \omega^{\prime \prime \prime}\right)\right) \equiv 0 \bmod 3$ and then $\nu_{\mathrm{p}}(\omega) \equiv 0 \bmod 3$ as asserted.

Case $2.3 k>2 l$. We shall use the following easily proved fact:

$$
\text { If } \gamma \in K_{3} \text { is } \mathfrak{p} \text {-integral, then } \mathfrak{p}^{n} \mid \gamma \text { implies } \mathfrak{p}^{n+2} \mid S_{3 / 1}(\gamma) \text {. }
$$

From (7.9) we have

$$
\varphi \tau^{-1}=\tau^{-1} S_{3 / 1}\left(\tau^{-1}\right)+S_{3 / 1}\left(\tau^{\prime}(\alpha-1)\right)-\tau\left(\alpha^{\prime \prime}-1\right)
$$

so that $\nu_{\mathfrak{p}}(\varphi)=2 l$ by $(7.10)$.

Suppose first that $l=1$. From (7.11) and (7.10),

$$
A=S_{3 / 1}\left(\varphi \tau^{-1}\right)=S_{3 / 1}\left(\tau^{-1}\right)^{2}+2 S_{3 / 1}\left(\tau^{\prime}(\alpha-1)\right) \equiv 0 \bmod 9 .
$$

In this case, we have $\nu_{\mathfrak{p}}(\varphi)=\nu_{\mathfrak{p}}(\psi)=\nu_{\mathfrak{p}}\left(\left(\omega, \omega^{\prime \prime \prime}\right)\right)=2$, whence $\nu_{\mathfrak{p}}(\omega) \not \equiv 0 \bmod 3$ as asserted.

Suppose next that $l \geqslant 2$. From $N_{6 / 2}(\alpha+\beta \sqrt{m})=1$ we have, by computing the coefficient of $\sqrt{m}$,

$$
S_{3 / 1}(\beta)+S_{3 / 1}\left(\left(\alpha \alpha^{\prime}-1\right) \beta^{\prime \prime}\right)+N_{3 / 1}(\beta) m=0 .
$$

In this equation, $\nu_{\mathfrak{p}}\left(N_{3 / 1}(\beta) m\right)=3 l$ and $\left(\alpha \alpha^{\prime}-1\right) \beta^{\prime \prime} \equiv 0 \bmod \mathfrak{p}^{3 l}$ so that $\nu_{\mathrm{p}}\left(S_{3 / 1}(\beta)\right)=3 l$ by $(7.10)$.

Denote $\beta=c_{0}+c_{1} \theta+c_{2} \theta^{\prime}$, where the $c_{i}$ 's are 3-integral rational numbers. Since $S_{3 / 1}(\beta)=3 c_{0}$, we have $3^{l-1} \| c_{0}$. As 3 is ramified in $K_{3} / \mathbf{Q}, \operatorname{Irr}(\theta, \mathbf{Q})=x^{3}-$ $\left(f_{3} / 3\right) x-f_{3} a / 27\left[13\right.$, p.9]. Clearly, $\theta \equiv-1 \bmod p$ and, further, $\nu_{p}\left(\theta-\theta^{\prime}\right)=2$ because

$$
N_{3 / 1}\left(\theta-\theta^{\prime}\right)^{2}=4\left(f_{3} / 3\right)^{3}-27\left(f_{3} a / 27\right)^{2}=f_{3}^{2}(b / 3)^{2},
$$

where $3^{1} \| b$. Writing

$$
\beta=c_{0}+\left(c_{1}+c_{2}\right) \theta-c_{2}\left(\theta-\theta^{\prime}\right)=c_{0}+\left(c_{1}+c_{2}\right) \theta^{\prime}+c_{1}\left(\theta-\theta^{\prime}\right)
$$

and taking into account that $\nu_{\mathfrak{p}}\left(c_{0}\right)=3 l-3>l=\nu_{\mathfrak{p}}(\beta)$, we find that there are two possibilities: either

$$
l \equiv 0 \bmod 3, \quad \nu_{\mathfrak{p}}\left(c_{1}+c_{2}\right)=l, \quad \nu_{\mathfrak{p}}\left(c_{i}\right) \geqslant l \quad(i=1,2),
$$

or

(7.13) $l \equiv 2 \bmod 3, \quad \nu_{\mathfrak{p}}\left(c_{1}\right)=\nu_{\mathfrak{p}}\left(c_{2}\right)=l-2, \quad c_{1}+c_{2} \equiv 0 \bmod \mathfrak{p}^{l+1}$.

In the case (7.12) we have $\nu_{p}(A) \equiv \nu_{p}(\varphi) \equiv 0 \bmod 3$ in the equation $A=\varphi^{2}-m \psi^{2}$ whence, either $\nu_{\mathfrak{p}}(\psi)>\nu_{\mathfrak{p}}(\varphi)$ or $\nu_{\mathfrak{p}}(\psi) \equiv 0 \bmod 3$, and the same argument as above gives $\nu_{\mathfrak{p}}(\omega) \equiv 0 \bmod 3$.

Suppose now that (7.13) holds. In the equation

$$
\psi \tau^{-1}=\tau^{\prime} \beta-\tau^{\prime \prime} \beta^{\prime}=\left(\tau^{\prime}-\tau^{\prime \prime}\right) \beta+\tau^{\prime \prime}\left(c_{1}+c_{2}\right)\left(\theta-\theta^{\prime}\right)+3 c_{2} \tau^{\prime \prime} \theta^{\prime}
$$

we have $\nu_{p}\left(3 c_{2} \tau^{\prime \prime} \theta^{\prime}\right)=l+1$ while the other terms on the right-hand side are divisible by a higher power of $\mathfrak{p}$. Hence $\nu_{\mathfrak{p}}(\psi)=l+1$ and the assertion $\nu_{\mathfrak{p}}(\omega) \equiv 0$ mod 3 follows by the standard argument. 
Case 3. $3 k=2 l$. If $\nu_{\mathrm{p}}(\varphi) \neq \nu_{\mathrm{p}}(\psi)$, then $\nu_{\mathrm{p}}(A)=\min \left\{\nu_{\mathrm{p}}\left(\varphi^{2}\right), \nu_{\mathrm{p}}\left(\psi^{2}\right)\right\}$ and $\nu_{\mathrm{p}}(\omega)$ $\equiv 0 \bmod 3$ as before. Suppose that $\nu_{\mathfrak{p}}(\varphi)=\nu_{\mathfrak{p}}(\psi)=n$, say. In this case we have $\nu_{p}(\omega) \equiv 0 \bmod 3$ iff $n \equiv 0 \bmod 3$.

For $N_{6 / 2}\left(U_{6}\right) \neq U_{2}$ and $h_{2} \equiv 0 \bmod 3$ it follows immediately from Lemma 3 and Theorem 6(i), that $\# \mathscr{C}_{6 / 2}=3$ if $f_{3} \mid 3 f_{2}$. Otherwise, one has to check that the prime factors of $f_{3}$ not dividing $f_{2}$ are inert in $K_{2} / \mathbf{Q}$ or that the conditions in (ii) and (iii) are not satisfied. This verification is most arduous in the very last case $p=3$, $3 k=2 l$, but this case seems to be scarce; we have encountered it only once.

8. Classes of $K_{3}$ in $K_{6}$. From [8, Ia, p. 92, Satz 12], we have

$$
\left[U_{R 3}: U_{6}^{1-\sigma^{3}}\right]=2^{4-q}
$$

where

$$
2^{q}=\left[N_{6 / 3}\left(U_{6}\right): U_{3}^{2}\right]= \begin{cases}8 & \text { if } N_{6 / 3}\left(U_{6}\right)=\left\langle-1, \tau, \tau^{\prime}\right\rangle=U_{3}, \\ 4 & \text { if } N_{6 / 3}\left(U_{6}\right)=\left\langle\tau, \tau^{\prime}\right\rangle, \\ 2 & \text { if } N_{6 / 3}\left(U_{6}\right)=\left\langle-1, \tau^{2}, \tau^{\prime 2}\right\rangle, \\ 1 & \text { if } N_{6 / 3}\left(U_{6}\right)=\left\langle\tau^{2}, \tau^{\prime 2}\right\rangle=U_{3}^{2} .\end{cases}
$$

LEMMA 4. A system of coset representatives of $U_{R 3}$ with respect to the subgroup $U_{6}^{1-\sigma^{3}}$ is

$$
\begin{array}{ll}
\left\{(-1)^{i} \mid i=0,1\right\} & \text { if } q=3, \\
\left\{(-1)^{i} \mu^{j} \mid i, j=0,1\right\} & \text { if } q=2, \\
\left\{(-1)^{i} \xi_{R}^{j} \xi_{R}^{\prime k} \mid i, j, k=0,1\right\} & \text { if } q=1, \\
\left\{(-1)^{i} \mu^{j} \xi_{R}^{k} \xi_{R}^{\prime l} \mid i, j, k, l=0,1\right\} & \text { if } q=0 .
\end{array}
$$

Proof. Suppose that there exists an $\varepsilon_{2} \in U_{2} \cap U_{6}^{1-\sigma^{3}}$. Write $\varepsilon_{2}=\varepsilon^{1-\sigma^{3}}$, where $\varepsilon \in U_{6}$. We have

$$
\left(\varepsilon^{4} \varepsilon_{2}^{-2}\right)^{1-\sigma^{3}}=\varepsilon_{2}^{2\left(1+\sigma^{3}\right)}=1,
$$

so that $\varepsilon^{4} \varepsilon_{2}^{-2} \in U_{3}$. From [13, p. 15, Theorem 2] we get $\varepsilon^{2} \varepsilon_{2}^{-1}=\varepsilon_{3}$, say, where $\varepsilon_{3} \in U_{3}$. From [13, p. 21, Theorem 9] we know that $\mathcal{O}_{6}$ has a system of fundamental units containing $\left\{\mu, \tau, \tau^{\prime}\right\}$. Since $\varepsilon_{2} \varepsilon_{3}$ is a square in $U_{6}, \pm \varepsilon_{2}$ is a square in $U_{2}$ and $\pm \varepsilon_{3}$ is a square in $U_{3}$. If, in particular, $\varepsilon_{2}=-1$, then $\varepsilon \in U_{3}$ which leads to the contradiction $-1=\varepsilon_{2}=\varepsilon^{1-\sigma^{3}}=1$. Since $\mu \in U_{R 3}$ if $q=2$, the assertion is plainly true for $q=3$ or 2 by (8.1) and (8.2).

Suppose, therefore, that $q=1$ or 0 so that $N_{6 / 3}\left(U_{6}\right)=\langle-1\rangle U_{3}^{2}$ or $U_{3}^{2}$. Let $\varepsilon_{2} \in U_{2}$ and $\varepsilon_{R} \in U_{R}$ and suppose that $\varepsilon_{2} \varepsilon_{R} \in U_{2} U_{R} \cap U_{6}^{1-\sigma^{3}}$. Denote $\varepsilon_{2} \varepsilon_{R}=\varepsilon^{1-\sigma^{3}}$ where $\varepsilon \in U_{6}$. As above, we have first

$$
\left(\varepsilon^{4} \varepsilon_{2}^{-2} \varepsilon_{R}^{-2}\right)^{1-\sigma^{3}}=1
$$

and then $\varepsilon^{2} \varepsilon_{2}^{-1} \varepsilon_{R}^{-1}=\varepsilon_{3}$, where $\varepsilon_{3} \in U_{3}$. Hence $\varepsilon_{3}^{2}=N_{6 / 3}(\varepsilon)^{2}$ so that

$$
\varepsilon_{3}= \pm N_{6 / 3}(\varepsilon) \in\langle-1\rangle N_{6 / 3}\left(U_{6}\right)=\langle-1\rangle U_{3}^{2} \text {. }
$$

Therefore, $\pm \varepsilon_{3}$ is a square in $U_{3}$. Further, $N_{6 / 2}(\varepsilon)^{2} \varepsilon_{2}^{-3}= \pm 1$ so that $\pm \varepsilon_{2}$ is a square in $U_{2}$. Finally, of course, $\pm \varepsilon_{R}$ is also a square in $U_{R}$. It is now easy to deduce the assertion from (8.1), (8.2) and the results in the first part of the proof. 
THEOREM 7. If $\varepsilon_{2} \in U_{2} \cap U_{R 3} \backslash U_{6}^{1-\sigma^{3}}$ and $\varepsilon_{R} \in U_{R} \cap U_{R 2}$, then $\varepsilon_{2} \varepsilon_{R} U_{6}^{1-\sigma^{3}} \notin$ $\operatorname{Im}\left(g_{3}\right)$. In particular, $\mathscr{C}_{6 / 3}=1$ if $\langle-1\rangle N_{6 / 3}\left(U_{6}\right)=U_{3}$.

Proof. The proof is similar in structure to that of Theorem 5. From Hilbert 90 we have $\varepsilon_{2} \varepsilon_{R}=\gamma^{1-\sigma^{3}}$ for some $\gamma \in \mathcal{O}_{6} \backslash\{0\}$. If $\varepsilon_{2} \varepsilon_{R} U_{6}^{1-\sigma^{3}} \in \operatorname{Im}\left(g_{3}\right)$, there exists an ideal $\mathfrak{c}$ of $\mathcal{O}_{3}$ such that $\gamma \mathcal{O}_{6}=\mathfrak{c} \mathcal{O}_{6}$. Taking norms, we have $N_{6 / 2}(\gamma) \mathcal{O}_{6}=N_{3 / 1}(\mathfrak{c}) \mathcal{O}_{6}=$ $c \mathcal{O}_{6}$, say, for some $c \in \mathbf{Z}$. Therefore, $N_{6 / 2}(\gamma)=c \varepsilon$, where $\varepsilon \in U_{2}$. Hence, $\varepsilon_{2}^{3}=$ $N_{6 / 2}\left(\varepsilon_{2} \varepsilon_{R}\right)=\varepsilon^{1-\sigma^{3}}$. It follows from $\varepsilon_{2} \in U_{R 3}$ that $\varepsilon_{2}^{2} \in U_{6}^{1-\sigma^{3}}$ and thus, also, $\varepsilon_{2} \in$ $U_{6}^{1-\sigma^{3}}$, contrary to the hypothesis. The rest of the assertion is clearly true.

From now on, in this section, we shall assume that $\langle-1\rangle N_{6 / 3}\left(U_{6}\right) \neq U_{3}$, i.e., $q=1$ or 0 . It is clear from Lemma 4 and Theorem 7, that $\operatorname{Im}\left(g_{3}\right)$ is either 1 or $\left\{\xi_{R}^{k} \xi_{R}^{\prime l} U_{6}^{1-\sigma^{3}} \mid k, l=0,1\right\}$, i.e., $\# \mathscr{C}_{6 / 3}=1$ or 4 . The latter alternative holds true for every such field $K_{6}$ with $h_{3} \equiv 0 \bmod 4$ up to $f_{6}<4000$.

A number satisfying Hilbert 90 for $\xi_{R}$ is $1+\xi_{R}$, i.e.,

$$
\xi_{R}=\left(1+\xi_{R}\right)^{1-\sigma^{3}} \text {. }
$$

Further,

$$
\left(1+\xi_{R}\right)^{1+\sigma^{3}}=2(\alpha+1) .
$$

From Lemma 1 and (8.3), we have

LEMMA 5. If $\langle-1\rangle N_{6 / 3}\left(U_{6}\right) \neq U_{3}$ and $h_{3} \equiv 0 \bmod 4$, then $\# \mathscr{C}_{6 / 3}=4$ if and only if $\nu_{\mathfrak{p}}\left(1+\xi_{R}\right) \equiv 0 \bmod 2$ for every prime ideal $\mathfrak{p}$ of $\mathcal{O}_{6}$ which is ramified in the extension $K_{6} / K_{3}$.

Our aim is to construct a criterion analogous to Theorem 6 which, however, will be of somewhat different type. For that purpose we need some auxiliary results.

LemMA 6. Suppose that $N_{6 / 2}\left(\xi_{R}\right)=1$ and that $4 \mid f_{2}$.

(i) If $\alpha \neq \equiv 1 \bmod 2$, then $a \equiv b \equiv 0 \bmod 2$ and $8+f_{2}$.

(ii) If $a \equiv b \equiv 1 \bmod 2$, then $2 \mid 1+\xi_{R}$ and $\left(\left(1+\xi_{R}\right) / 2,2\right)=1$.

Proof. We shall use the following well-known fact:

The prime 2 is inert in $K_{3} / \mathbf{Q}$ if and only if $a \equiv b \equiv 1 \bmod 2$.

We prove (i) first. If $2 \mid m \beta$ it follows from $(\alpha-1)(\alpha+1)=m \beta^{2}$ that $\alpha \equiv 1 \mathrm{mod}$ 2. We shall therefore assume that $m \equiv 3 \bmod 4, a \equiv b \equiv 1 \bmod 2$ (i.e., 2 is inert in $\left.K_{3} / \mathbf{Q}\right), 2+\beta$, and show that these assumptions lead to a contradiction.

Since $\left\{1, \theta, \theta^{\prime}\right\}$ is an integral basis for $\mathcal{O}_{3}$ and $b$ is odd, it follows from the expression of $\theta^{\prime}\left[13\right.$, pp. 8-9], that $\left\{1, \theta, \theta^{2}\right\}$ is a local integral basis at the prime 2 . Replacing $\theta$ by one of its conjugates if need be, we may assume that $\beta \equiv h+k \theta$ $\bmod 2$, where $h, k \in\{0,1\}$. Let $\alpha \equiv q+r \theta+s \theta^{2} \bmod 2$, where $q, r, s \in\{0,1\}$.

Suppose first that $s=1$. We have $\theta^{4} \equiv e+\theta+\theta^{2} \bmod 2$, where $e=1$ if $3+f_{3}$ and $e=0$ if $3 \mid f_{3}$. From $\alpha^{2}-m \beta^{2}=1$ we get

$$
h+q+e+\theta+(k+r+1) \theta^{2} \equiv 1 \bmod 2,
$$

a contradiction. Hence $s=0$ and we have similarly

$$
q^{2}-m h^{2}+2(q r+h k) \theta+\left(r^{2}-m k^{2}\right) \theta^{2} \equiv 1 \bmod 4 .
$$

In particular, $r^{2}-m k^{2} \equiv 0 \bmod 4$ whence $r=k=0$ because of the assumption $m \equiv 3 \bmod 4$. Since $2+\beta$, we have $h=1$ so that $q=0$ by (8.6). Then $2 \mid \alpha$, so that $N_{6 / 2}\left(\xi_{R}\right)=1$ implies $m N_{3 / 1}(\beta) \sqrt{m} \equiv 1 \bmod 2$, a contradiction because $m \equiv 3$ $\bmod 4$. This completes the proof of (i). 
To prove (ii) we still assume that $a \equiv b \equiv 1 \bmod 2$. From (i) we know that $\alpha \equiv 1$ mod 2. From (8.4) we have

$$
\operatorname{Irr}\left(1+\xi_{R}, K_{3}\right)=x^{2}-2(\alpha+1) x+2(\alpha+1)
$$

so that $2 \mid 1+\xi_{R}$. Let $\mathfrak{p}$ be a prime ideal of $\mathcal{O}_{6}$ dividing 2 . Then by (8.5), 20 $\mathcal{O}_{6}=\mathfrak{p}^{2}$. If $\left(\left(1+\xi_{R}\right) / 2,2\right) \neq 1$, then $4 \mid \alpha+1$. From $m \beta^{2}=(\alpha-1)(\alpha+1)$, we obtain $8 \mid m \beta^{2}$ so that either $m \equiv \beta \equiv 0 \bmod 2$ or $\beta \equiv 0 \bmod 4$. In both cases,

$$
1=N_{6 / 2}\left(\xi_{R}\right) \equiv \alpha \alpha^{\prime} \alpha^{\prime \prime} \equiv-1 \bmod \mathfrak{p}^{3}
$$

which is impossible.

LEMMA 7. Suppose that $N_{6 / 2}\left(\xi_{R}\right)=1$. Let $\mathfrak{p}$ be a prime ideal of $\mathcal{O}_{6}$ which is ramified in $K_{6} / K_{3}$. Write $\mathfrak{p} \cap \mathbf{Z}=p \mathbf{Z}$. If $p$ is ramified or inert in $K_{3} / \mathbf{Q}$, then

$$
\nu_{p}\left(1+\xi_{R}\right)= \begin{cases}0 & \text { if } p \neq 2 \\ 2 & \text { if } p=2\end{cases}
$$

Proof. If $p=2$ then $p$ is inert in $K_{3} / \mathbf{Q}$ and the result follows immediately from Lemma 6(ii). Suppose that $p \neq 2$. Then $\beta \sqrt{m}$ is a $p$-integer divisible by $\mathfrak{p}$ because $\nu_{p}(\beta)$ is even and $\nu_{p}(\sqrt{m})$ is odd. Suppose that, contrary to the assertion, $\alpha \equiv-1$ $\bmod \mathfrak{p}$. It would follow that $1=N_{6 / 2}\left(\xi_{R}\right) \equiv \alpha \alpha^{\prime} \alpha^{\prime \prime} \equiv-1 \bmod \mathfrak{p}$, a contradiction.

We are now ready to state the main criterion.

ThEOREM 8. Let $\xi_{R}=\alpha+\beta \sqrt{m}$, where $\alpha, \beta \in K_{3}$. Suppose that $N_{6 / 2}\left(\xi_{R}\right)=1$. Denote

$$
P=2^{\lambda} \prod p
$$

where the product is to be taken over all odd primes $p$ dividing $f_{2}$ which split in $K_{3}$, and

$$
\lambda= \begin{cases}2 & \text { if } a \equiv b \equiv 0 \bmod 2 \text { and } m \equiv 2 \bmod 4 \\ 1 & \text { if } a \equiv b \equiv 0 \bmod 2 \text { and } m \equiv 3 \bmod 4 \\ 0 & \text { otherwise. }\end{cases}
$$

Then the following conditions are equivalent:

(i) We have $\nu_{\mathfrak{p}}\left(1+\xi_{R}\right) \equiv 0$ mod 2 for every prime ideal $\mathfrak{p}$ of $\mathcal{O}_{6}$ which is ramified in $K_{6} / K_{3}$.

(ii) The number $\alpha$ is congruent to a rational integer $\bmod P$.

Proof. Let $\mathfrak{p}$ be a prime ideal of $\mathcal{O}_{6}$ which is ramified in $K_{6} / K_{3}$. Write $\mathfrak{p} \cap \mathbf{Z}=p \mathbf{Z}$. If $p$ is ramified or inert in $K_{3} / \mathbf{Q}$, then $\nu_{\mathrm{p}}\left(1+\xi_{R}\right) \equiv 0 \bmod 2$ by Lemma 7 , and, on the other hand, no condition upon $\alpha \bmod p$ is imposed in (ii). It is therefore enough to assume that $p$ splits in $K_{3}$.

We consider first the case $p \neq 2$. As in the proof of Lemma 7 we have $\beta \sqrt{m} \equiv$ $\beta^{\prime} \sqrt{m} \equiv \beta^{\prime \prime} \sqrt{m} \equiv 0 \bmod \mathfrak{p}$. If $\alpha \equiv c \bmod p$, where $c \in \mathbf{Z}$, it follows from $\alpha^{2}-m \beta^{2}$ $=1$ that $c^{2} \equiv 1 \bmod p$, and from $1=N_{6 / 2}\left(\xi_{R}\right) \equiv \alpha \alpha^{\prime} \alpha^{\prime \prime} \bmod p$ that $c^{3} \equiv 1 \bmod p$. Thus $c \equiv 1 \bmod p$ and $2(\alpha+1) \equiv 4 \bmod p$. Hence, none of the conjugates of $p$ divides $1+\xi_{R}$ by (8.4). Suppose, on the contrary, that $\alpha$ is not congruent to a rational integer $\bmod p$. It follows from $(\alpha-1)(\alpha+1)=m \beta^{2} \equiv 0 \bmod p$ that for some prime ideal $\mathfrak{p}$ of $\mathcal{O}_{6}$ dividing $p$ we must have $\mathfrak{p} \mid \alpha+1$, because otherwise $\alpha \equiv 1$ mod $p$ contrary to the hypothesis. Furthermore, by (8.4),

$$
2 \nu_{\mathfrak{p}}\left(1+\xi_{R}\right)=\nu_{\mathfrak{p}}(\alpha+1)=\nu_{\mathfrak{p}}(m)+2 \nu_{\mathfrak{p}}(\beta),
$$

implying that $\nu_{\mathfrak{p}}\left(1+\xi_{R}\right)$ is odd. We have thus proved the equivalence (i) and (ii) locally at the prime $p$. 
Consider next the case $p=2$. We have $a \equiv b \equiv 0 \bmod 2$ by (8.5) and $m \equiv 2$ or 3 mod 4 because 2 is ramified in $K_{2} / \mathbf{Q}$. First let $m \equiv 2 \bmod 4$. From Lemma 6(i) we have $\alpha \equiv 1 \bmod 2$. We write

$$
((\alpha-1) / 2)((\alpha+1) / 2)=m(\beta / 2)^{2}
$$

because, obviously, $2 \mid \beta$. If $\alpha \equiv c \bmod 4$ for some $c \in \mathbf{Z}$, one can conclude in the same way as in the first part of the proof that $c \equiv 1 \bmod 4$. Then, $((\alpha+1) / 2,2)=1$ so that from (8.4),

$$
2 \nu_{p}\left(1+\xi_{R}\right)=\nu_{p}(4)=4
$$

On the other hand, if $\alpha$ is not congruent to a rational integer mod 4 , it follows from (8.7) that for some prime ideal factor $p$ of 2 in $\mathcal{O}_{6}$ we must have $(\alpha+1) / 2 \equiv 0$ mod $\mathfrak{p},(\alpha-1) / 2 \not \equiv 0$ mod $\mathfrak{p}$. For this $\mathfrak{p}$ we have, by (8.4) and (8.7),

$$
2 \nu_{\mathfrak{p}}\left(1+\xi_{R}\right)=\nu_{\mathfrak{p}}(4)+\nu_{\mathfrak{p}}((\alpha+1) / 2)=4+\nu_{\mathfrak{p}}(m)+2 \nu_{\mathfrak{p}}(\beta / 2) \text {, }
$$

whence

$$
\nu_{\mathfrak{p}}\left(1+\xi_{R}\right)=3+\nu_{\mathfrak{p}}(\beta / 2) \equiv 1 \bmod 2 .
$$

This proves the equivalence of (i) and (ii) at the prime 2 in the case $m \equiv 2 \bmod 4$.

Suppose, finally, that $m \equiv 3 \bmod 4$. If $\alpha \equiv c \bmod 2$ for some $c \in \mathbf{Z}$, the same argument as at the end of the proof of Lemma 6(i) shows that $c$ cannot be even. Hence, $\alpha \equiv 1 \bmod 2$ and the numbers appearing in (8.7) are algebraic integers. For any $\mathfrak{p}$ dividing 2 we have $\nu_{\mathfrak{p}}((\alpha+1) / 2)=0$ or $2 \nu_{\mathfrak{p}}(\beta / 2)$, both of which are $\equiv 0$ mod 4. From $(8.4), \nu_{p}\left(1+\xi_{R}\right) \equiv 0 \bmod 2$.

If $\alpha$ is not congruent to a rational integer mod 2 , then for some $\mathfrak{p}$ dividing 2 , we have $\mathfrak{p}+\alpha+1$. For this $\mathfrak{p}, \nu_{\mathfrak{p}}\left(1+\xi_{R}\right)=1$ by (8.4). This concludes the proof of Theorem 8 .

If $\langle-1\rangle N_{6 / 3}\left(U_{6}\right) \neq U_{3}$ and $h_{3} \equiv 0 \bmod 4$, it follows from Lemma 5 and Theorem 8 that $\# \mathscr{C}_{6 / 3}=4$ in the case $f_{2} \mid f_{3}$. Otherwise, Theorem 8 provides a very convenient tool for establishing the truth of that conjectural fact.

9. Tables and Statistics. The table containing the 12 cases missing from [13] and the 1743 fields with $2021<f_{6}<4000$ has been deposited in the Mathematics of Computation's UMT-depository. The data in the table are listed in the same format as in [13] with the following slight changes. Firstly, no distinction is made between the cases $r 1$ and $r 2$ when $h_{R}=7$. Secondly, let $\xi_{*}$ be any of the units whose coordinates multiplied by $k[13$, p. 68] are listed in the table. Here $*$ stands for any of the letters $A, R, B, C$. Denote $k \xi_{*}=c_{0}+c_{1} \theta+c_{2} \theta^{\prime}+\left(d_{0}+d_{1} \theta+d_{2} \theta^{\prime}\right) \sqrt{m}$, where $c_{i}, d_{i} \in \mathbf{Z}$. These numbers are arranged in the form of the matrix

$$
\left(\begin{array}{lll}
c_{0} & c_{1} & c_{2} \\
d_{0} & d_{1} & d_{2}
\end{array}\right)
$$

as in [13] if there is enough space for that. If not, they are written one underneath the other in the natural order $c_{0}, c_{1}, c_{2}, d_{0}, d_{1}, d_{2}$.

In each of the 12 cases missing from [13] the relative class number $h_{R}=1$. The corresponding values of $f_{6}$ are $997,1021,1093,1561,1597,1657,1753,1777,1801$, $1933,1981,2017$. Of these, $1561=7 \times 223,1981=7 \times 283$, and the other 10 numbers are primes. 
TABLE 1

\begin{tabular}{rrrrrrrrrrrr}
\hline$f_{6}$ & $h_{R}$ & $h_{2}$ & $h_{3}$ & $f_{6}$ & $h_{R}$ & $h_{2}$ & $h_{3}$ & $f_{6}$ & $h_{R}$ & $h_{2}$ & $h_{3}$ \\
\hline 229 & 1 & 3 & 1 & 277 & 1 & 1 & 4 & 313 & 1 & 1 & 7 \\
349 & 4 & 1 & 4 & 397 & 1 & 1 & 4 & 577 & 1 & 7 & 1 \\
709 & 4 & 1 & 4 & 733 & 1 & 3 & 1 & 853 & 1 & 1 & 4 \\
877 & 7 & 1 & 7 & 937 & 4 & 1 & 4 & 1009 & 1 & 7 & 4 \\
1069 & 7 & 1 & 1 & 1093 & 1 & 5 & 1 & 1129 & 1 & 9 & 7 \\
1297 & 1 & 11 & 1 & 1381 & 7 & 1 & 1 & 1429 & 1 & 5 & 1 \\
1489 & 1 & 3 & 19 & 1777 & 1 & 1 & 16 & 1789 & 1 & 1 & 4 \\
2029 & 1 & 7 & 1 & 2089 & 3 & 3 & 1 & 2437 & 1 & 1 & 7 \\
2557 & 7 & 3 & 7 & 2677 & 1 & 3 & 1 & 2689 & 1 & 1 & 4 \\
2713 & 1 & 3 & 1 & 2797 & 1 & 1 & 4 & 2857 & 1 & 3 & 1 \\
2917 & 7 & 3 & 1 & 3037 & 1 & 1 & 4 & 3121 & 1 & 5 & 1 \\
3181 & 1 & 5 & 1 & 3217 & 1 & 1 & 7 & 3229 & 3 & 3 & 1 \\
3253 & 1 & 5 & 1 & 3313 & 7 & 1 & 19 & 3469 & 13 & 1 & 1 \\
3517 & 1 & 1 & 4 & 3529 & 1 & 1 & 19 & 3877 & 1 & 3 & 1 \\
3889 & 1 & 3 & 1 & & & & & & & & \\
\hline
\end{tabular}

TABLE 2

\begin{tabular}{clrr}
\hline Type & & $h_{R}$ & No \\
\hline 1 & $\xi_{R}^{2}= \pm \xi_{0}$ & 4 & 42 \\
2 & $\xi_{R}^{3}=\xi_{0}$ & 9 & 1 \\
3 & $\xi_{R}^{5}=\xi_{0}$ & 25 & 1 \\
4 & $\xi_{R}^{3}=\xi_{0} \xi_{0}^{\prime}$ & 3 & 40 \\
5 & $\xi_{R}^{7}=\xi_{0}^{2} \xi_{0}^{\prime}$ or $\xi_{0} \xi_{0}^{\prime 2}$ & 7 & 72 \\
6 & $\xi_{R}^{13}=\xi_{0}^{3} \xi_{0}^{\prime}$ or $\xi_{0} \xi_{0}^{\prime 3}$ & 13 & 17 \\
7 & $\xi_{R}^{19}=\xi_{0}^{3} \xi_{0}^{\prime 2}$ & 19 & 2 \\
8 & $\xi_{R}^{61}=\xi_{0}^{5} \xi_{0}^{\prime 4}$ & 61 & 1 \\
9 & $\xi_{R}=\xi_{0}, \xi_{B}$ exists & 3 & 94 \\
10 & $\xi_{R}=\xi_{0}, \xi_{C}$ exists & 4 & 130 \\
11 & $\xi_{R}^{2}=\xi_{0}, \xi_{B}$ exists & 12 & 1 \\
12 & $\xi_{R}^{3}=\xi_{0} \xi_{0}^{\prime}, \xi_{B}$ exists & 9 & 6 \\
13 & $\xi_{R}^{7}=\xi_{0}^{2} \xi_{0}^{\prime}, \xi_{B}$ exists & 21 & 1 \\
14 & $\xi_{R}^{2}= \pm \xi_{0}, \xi_{C}$ exists & 16 & 6 \\
15 & $\xi_{R}^{3}=\xi_{0} \xi_{0}^{\prime}, \xi_{C}$ exists & 12 & 2 \\
16 & $\xi_{R}=\xi_{0}, \xi_{B}$ and $\xi_{C}$ exist & 12 & 3 \\
\hline
\end{tabular}

There are 131 prime sextic conductors $f_{6}$ less that 4000 . They are of particular interest for a number of reasons, especially because of the following well-known fact: If $f_{6}$ is prime and $L$ is any subfield of $C\left(f_{6}\right)$ containing $K_{6}$, then the class number of $L$ is divisible by $h_{6}$. For an interesting historical remark concerning a closely related result see [1, p. 219 , Footnote 3$]$. There are exactly 43 prime sextic conductors $f_{6}$ less than 4000 such that the class number $h_{6}=h_{R} h_{2} h_{3}>1$. These conductors and the class numbers $h_{R}, h_{2}, h_{3}$ are listed in Table 1.

We note that all statistics we give in this paper concern the total range $1<f_{6}<$ 4000 and thus include the statistics in [13]. There are altogether 419 fields $K_{6}$ with $f_{6}<4000$ such that $h_{R}>1$. These fields can be divided into 16 different types depending on the expression of $\xi_{R}$ in terms of $\xi_{0}$ and $\xi_{0}^{\prime}$ and on the existence of $\xi_{B}$ or $\xi_{C}$. A similar division was exercised in [13] but only 8 types emerged there. In Table 2 we give for each type the relative class number $h_{R}$ and the number of fields 
TABLE 3

\begin{tabular}{rrrrrrrrrrrr}
\hline$f_{6}$ & $f_{2}$ & $f_{3}$ & $a$ & $b$ & Type & $f_{6}$ & $f_{2}$ & $f_{3}$ & $a$ & $b$ & Type \\
\hline 995 & 5 & 199 & 11 & 15 & 16 & 1143 & 381 & 1143 & -3 & 39 & 14 \\
1548 & 172 & 387 & -39 & 3 & 2 & 2077 & 2077 & 2077 & -91 & 3 & 14 \\
2135 & 305 & 427 & -40 & 6 & 16 & 2289 & 21 & 763 & 53 & 9 & 15 \\
2428 & 2428 & 607 & -49 & 3 & 14 & 2439 & 813 & 2439 & -3 & 57 & 3 \\
2669 & 17 & 157 & 14 & 12 & 13 & 2812 & 76 & 703 & -25 & 27 & 14 \\
2844 & 316 & 711 & -12 & 30 & 16 & 2869 & 2869 & 2869 & 107 & 3 & 7 \\
2921 & 2921 & 127 & 20 & 6 & 8 & 2945 & 5 & 589 & 41 & 15 & 15 \\
3003 & 429 & 91 & 11 & 9 & 11 & 3052 & 3052 & 763 & -55 & 3 & 14 \\
3155 & 5 & 631 & -43 & 15 & 14 & 3339 & 53 & 63 & 15 & 3 & 12 \\
3339 & 1113 & 63 & -12 & 6 & 7 & 3432 & 264 & 13 & 5 & 3 & 12 \\
3572 & 188 & 19 & -7 & 3 & 12 & 3708 & 412 & 9 & -3 & 3 & 12 \\
3981 & 3981 & 1327 & -4 & 42 & 12 & 3983 & 569 & 7 & -1 & 3 & 12 \\
\hline
\end{tabular}

TABLE 4

\begin{tabular}{|c|c|c|c|c|c|c|c|c|c|c|}
\hline & \multicolumn{5}{|c|}{$\chi_{6}$ decomposable } & \multicolumn{5}{|c|}{$\chi_{6}$ nondecomposable } \\
\hline & 3 & 4 & 5 & 6 & $\Sigma$ & 3 & 4 & 5 & 6 & $\Sigma$ \\
\hline $1<f_{6} \leqslant 1000$ & 60 & 45 & 155 & 146 & 406 & 20 & 3 & 106 & 33 & 162 \\
\hline $1000<f_{6} \leqslant 2000$ & 131 & 57 & 252 & 168 & 608 & 26 & 3 & 89 & 31 & 149 \\
\hline $2000<f_{6} \leqslant 3000$ & 155 & 61 & 303 & 208 & 727 & 28 & 2 & 77 & 27 & 134 \\
\hline $300 c<f_{6} \leqslant 4000$ & 156 & 79 & 301 & 215 & 751 & 21 & 2 & 90 & 30 & 143 \\
\hline$\Sigma$ & 502 & 242 & 1011 & 737 & 2492 & 95 & 10 & 362 & 121 & 588 \\
\hline
\end{tabular}

belonging to that type. In Table 3 we list the parameters $f_{6}, f_{2}, f_{3}, a, b$ of the fields belonging to the less frequent and perhaps more interesting types $2,3,7,8,11-16$.

Statistics referring to the signature rank $\mathrm{Sr}$ of the unit group $U_{6}$ are given in Table 4 in the decomposable and nondecomposable cases separately. The numbers at the top of the columns are the values of $\mathrm{Sr}$.

There are 14 fields $K_{6}$ with $f_{6}<4000$ such that the norm-positive cubic units in $U_{3}$ are totally positive. As noted in [13, p. 70] it is of importance to be able to recognize these cases. These fields and the class numbers $h_{R}, h_{2}, h_{3}$ are listed below.

\begin{tabular}{rrrrrrrr}
$f_{6}$ & $f_{2}$ & $f_{3}$ & $a$ & $b$ & $h_{R}$ & $h_{2}$ & $h_{3}$ \\
\hline 703 & 37 & 703 & -25 & 27 & 1 & 1 & 12 \\
711 & 237 & 711 & -12 & 30 & 1 & 1 & 12 \\
1009 & 1009 & 1009 & -43 & 27 & 1 & 7 & 4 \\
2109 & 57 & 703 & -25 & 27 & 1 & 1 & 12 \\
2109 & 2109 & 703 & -25 & 27 & 1 & 2 & 12 \\
2812 & 76 & 703 & -25 & 27 & 16 & 1 & 12 \\
2812 & 2812 & 703 & -25 & 27 & 1 & 2 & 12 \\
2844 & 12 & 711 & -12 & 30 & 4 & 1 & 12 \\
2844 & 316 & 711 & -12 & 30 & 12 & 3 & 12 \\
3193 & 3193 & 3193 & -55 & 57 & 4 & 1 & 12 \\
3515 & 5 & 703 & -25 & 27 & 1 & 1 & 12 \\
3515 & 185 & 703 & -25 & 27 & 1 & 2 & 12 \\
3555 & 5 & 711 & -12 & 30 & 1 & 1 & 12 \\
3555 & 1185 & 711 & -12 & 30 & 1 & 2 & 12
\end{tabular}


The unit index $Q_{K}$ is not listed in the tables but it is easily determined by means of the following two rules:

$$
\begin{gathered}
N_{6 / 2}\left(U_{6}\right) \neq U_{2} \Leftrightarrow 3 \mid w \text { and } \xi_{B} \text { does not exist, } \\
\langle-1\rangle N_{6 / 3}\left(U_{6}\right) \neq U_{3} \Leftrightarrow 2|u, 2| v \text { and } \xi_{C} \text { does not exist. }
\end{gathered}
$$

The distribution of the values of $Q_{K}$ is as follows:

\begin{tabular}{|r|r|r|r|r|r} 
& 12 & 3 & 4 & 1 & $\Sigma$ \\
\hline $1<f_{6} \leqslant 1000$ & 347 & 100 & 93 & 28 & 568 \\
$1000<f_{6} \leqslant 2000$ & 394 & 152 & 146 & 65 & 757 \\
$2000<f_{6} \leqslant 3000$ & 443 & 179 & 175 & 64 & 861 \\
$3000<f_{6} \leqslant 4000$ & 462 & 185 & 175 & 72 & 894 \\
\hline$\Sigma$ & 1646 & 616 & 589 & 229 & 3080
\end{tabular}

In conclusion, we would like to draw attention to a rather frequently occurring connection between the relative class numbers $h_{R}$ of distinct fields $K_{6}$ having the same conductor $f_{6}$ and the same subfield $K_{2}$ or $K_{3}$. Consider values of $f$ such that for $n=2$ or 3 there is a quadratic (resp. cubic) field $K_{n}$ contained in more than one sextic field $K_{6}$ having conductor $f_{6}=f$ at least one of these fields $K_{6}$ having relative class number $h_{R}$ divisible by $6 / n$. Let $P(f, n)$ denote the following property:

For any given quadratic (resp. cubic) field $K_{n}$ the relative class numbers $h_{R}$ of the sextic fields $K_{6}$ having conductor $f_{6}=f$ and containing $K_{n}$ are either all prime to $6 / n$ or all divisible by $6 / n$.

The values of $f$ satisfying the preliminary requirement above are the following ones:

$$
n=2, \quad P(f, 2) \text { is true }
$$

793, 981, 1027, 1548, 1629, 1736, 2135, 2163, 2184, 2289, 2331, $2405,2412,2639,2701,2844,2945,2983,3003,3007,3033,3339$, $3416,3492,3573,3601,3708,3892,3999$

$$
n=2, \quad P(f, 2) \text { is false }
$$

469, 1603, 1708, 1957, 2977, 3303

$$
n=3, \quad P(f, 3) \text { is true }
$$

651, 732, 741, 1073, 1221, 1281, 1449, 1464, 1533, 1628, 1729, $1833,1935,2013,2044,2135,2289,2444,2604,2660,2821,2844$, $2849,2945,2964,3003,3059,3069,3081,3108,3233,3255,3256$, $3445,3464,3465,3477,3601,3627,3660,3705,3717,3784$

$$
n=3, \quad P(f, 3) \text { is false }
$$

248, 744, 936, 1064, 1240, 1368, 1736, 2072, 2456, 2709, 2728, $2812,3052,3192,3224,3512,3720,3752,3913,3992,3999$ 
1. N. C. Ankeny, S. Chowla \& H. Hasse, "On the class-number of the maximal real subfield of a cyclotomic field," J. Reine Angew. Math., v. 217, 1965, pp. 217-220. MR 30 \#3078.

2. Veikko Ennola \& Reino Turunen, “On totally real cubic fields," Math. Comp., v. 44, 1985, pp. $495-518$.

3. Veikko Ennola \& Reino Turunen, “On cyclic cubic fields," Math. Comp., v. 45, 1985, pp. $585-589$.

4. H. J. Godwin, "The calculation of large units in cyclic cubic fields," J. Reine Angew. Math., v. 338, 1983, pp. 216-220. MR 84e:12003.

5. MARIE - Nicole Gras, "Méthodes et algorithmes pour le calcul numérique du nombre de classes et des unités des extensions cubiques cycliques de Q," J. Reine Angew. Math., v. 277, 1975, pp. 89-116. MR 52 \#10675.

6. Marie-Nicole Gras, "Table numérique du nombre de classes et des unités des extensions cycliques réelles de degré 4 de Q,” Publ. Math. Univ. Besançon, 1977/78, fasc. 2.

7. Marie-Nicole Gras, “Classes et unités des extensions cycliques réelles de degré 4 de $\mathbf{Q}$," Ann. Inst. Fourier (Grenoble), v. 29, no. 1, 1979, pp. 107-124. MR 81f: 12003.

8. Helmut HASSE, Bericht über neuere Untersuchungen und Probleme aus der Theorie der algebraischen Zahlkörper. Teil I: Klassenkörpertheorie. Teil Ia: Beweise zu Teil I. Teil II: Reziprozitätsgesetz, Dritte Auflage, Physica-Verlag, Würzburg-Vienna, 1970. MR 42 \#1795.

9. Helmut Hasse, "Arithmetische Bestimmung von Grundeinheit und Klassenzahl in zyklischen kubischen und biquadratischen Zahlkörpern," Abh. Deutsch. Akad. Wiss. Berlin, Math.-Nat. Kl., 1948, No 2, 1950. MR 11, 503.

10. Helmut HaSSE, Über die Klassenzahl abelscher Zahlkörper, Akademie-Verlag, Berlin, 1952. MR 14, 141.

11. H. W. LEOPOLDT, “Über Einheitengruppe und Klassenzahl reeller abelscher Zahlkörper,” $A b h$. Deutsch. Akad. Wiss. Berlin, Math.-Nat. Kl., 1953, No 2, 1954. MR 16, 799.

12. A. MATUlJaUSKAS, "Integral representations of the cyclic group of order six," Litovsk. Mat. Sb., v. 2, no. 2, 1962, pp. 149-157. (Russian) MR 27 \#835.

13. SiRPa Mäki, The Determination of Units in Real Cyclic Sextic Fields, Lecture Notes in Math., vol. 797, Springer-Verlag, Berlin and New York, 1980. MR 82a: 12004.

14. Trygve Nagell, "Sur un type particulier d'unités algébriques," Ark. Mat., v. 8, 1969, pp. 163-184. MR 42 \#3064.

15. J. H. OpPenheIm, Integral Representations of Cyclic Groups of Square-Free Order, Ph.D. Thesis, Univ. of Illinois, Urbana, Ill., 1962. 